\title{
Paths of Real Roots for Odd-Degree Polynomials with Parameters
}

\author{
Junxiang Xu $\mathbb{1}$ \\ School of Mathematics, Southeast University, Nanjing 210096, China \\ Correspondence should be addressed to Junxiang Xu; xujun@seu.edu.cn
}

Received 8 July 2020; Accepted 30 September 2020; Published 9 November 2020

Academic Editor: Victor Kovtunenko

Copyright ( 2020 Junxiang Xu. This is an open access article distributed under the Creative Commons Attribution License, which permits unrestricted use, distribution, and reproduction in any medium, provided the original work is properly cited.

In this paper, we construct a connected path of real roots of odd-degree real polynomials depending on parameters continuously, which joins with all parameters. Moreover, most of the points on the path are odd-multiple real roots except for at most countable even-multiple real roots. Furthermore, some geometrical features of the path can be seen easily from the construction.

\section{Problem}

The problem of real roots of approximating real polynomials (small perturbations of real polynomials) with parameters is not only an elementary problem in theory but also a very important one in application. On the one hand, it is very interesting to understand the topological structure of the set of real roots of (approximating) real polynomials with parameters. On the other hand, it can arise in the study of small perturbations of degenerate systems such as in [1-5]. For example, when we consider the persistence of degenerate elliptic lower dimensional invariant tori in Hamiltonian systems, we must solve real roots of approximating real polynomials with a small parameter; moreover, we require to know how these real roots depend on the small parameter, which is very important for us to deal with the problem of small divisors in the KAM theory. It is well known that the small divisor condition in the KAM theory is usually guaranteed by adjusting some parameters. For this purpose, it is necessary to know the dependence of the frequencies on the parameter. This problem is the main motivation of this paper. We will understand it more precisely in a forthcoming paper, in which we will prove the persistence of degenerate elliptic lower dimensional invariant tori in Hamiltonian systems by the results obtained in this paper.

If an approximating polynomial depends on a small parameter, by some division theorems in [6-10], it can decompose into a product of a polynomial and a nonzero factor. Thus, in this paper, we mainly consider odd-degree real polynomials. We should first note that by the Leray-
Schauder continuation theorem $[11,12]$, we can also have a path in the set of real roots of $f$ joining with all parameters. However, we have no more information about the geometrical structure of the path. This is because the continuation theorem does not require too much smoothness assumption. In this paper, we construct a path in the set of all the real roots which joins with all parameters; moreover, it includes at most countable even-multiple real roots. From the proof, we can know more information about the geometrical structure of the path. This is what we are interested in.

\section{Main Results}

Consider a real polynomial of degree $2 d+1$ :

$$
f(\xi, x)=x^{2 d+1}+\sum_{j=1}^{2 d+1} c_{j}(\xi) x^{2 d+1-j}
$$

where the coefficients $\left\{c_{j}, 1 \leq j \leq 2 d+1\right\}$ depend continuously on a parameter $\xi \in[a, b]$. It is easy to know that $f$ has at least one real root for every $\xi$. Moreover, it is well known (see [13]) that there exist $2 d+1$ complex roots continuously depending on $\xi$, which are also called $2 d+1$ continuous bifurcations of roots. More precisely, we have the following result.

Lemma 1 (see [12]). Consider an $m$-degree polynomial

$$
f(\xi, x)=x^{m}+\sum_{1 \leq j \leq m} c_{j}(\xi) x^{m-j},
$$


where $\left\{c_{j}\right\}$ are continuous on $[a, b]$. Then, there exist $m$ continuous complex-valued functions.

$$
x_{1}(\xi), x_{2}(\xi), \cdots x_{m}(\xi) \quad \xi \in[a, b],
$$

such that the set

$$
\left\{\left(\xi, x_{j}(\xi)\right) \mid \xi \in[a, b], j=1,2, \cdots m\right\},
$$

consists of all roots of $f(\xi, x)$ for $\xi \in[a, b]$.

However, it is possible that there does not exist any continuous bifurcation of real roots for $\xi$ on $[a, b]$. But by our observation, we find that for a family of parameterized odddegree real polynomial $f(\xi, x)$, there exists a connected path of real roots on the $(\xi, x)$-plane, which joins with two oddmultiple real roots on the lines $\xi=a$ and $\xi=b$, respectively. This shows that the set of real roots of $f(\xi, x)$ contains a path which can connect all parameters $\xi \in[a, b]$. Moreover, the path includes at most countable even-multiple real roots; in other words, almost everywhere, points on the path are odd-multiple real roots.

To state our result, we first introduce some notations and definitions.

If $x_{0}$ is an $m$-multiple real root of $f\left(\xi_{0}, x\right)$, that is,

$$
f_{x}^{(k)}\left(\xi_{0}, x_{0}\right)=0, k=0,1, \cdots, m-1, f_{x}^{(m)}\left(\xi_{0}, x_{0}\right) \neq 0,
$$

we call $\left(\xi_{0}, x_{0}\right)$ an $m$-multiple real root of $f$.

Let

$$
a_{f,[a, b]}=\{(\xi, x) \in[a, b] \times \mathbb{R} \mid f(\xi, x)=0, \xi \in[a, b]\},
$$

be the set which consists of all real roots of $f$. Sometimes, we write $A=A_{f},[a, b]$ for simplicity if $f$ and $[a, b]$ are fixed. Let

$$
\begin{aligned}
A^{\text {odd }} & =\{P \in A \mid P \text { is an odd-multiple real root }\}, \\
A^{\text {even }} & =\{P \in A \mid P \text { is an even-multiple real root }\} .
\end{aligned}
$$

Let

$$
A(\xi)=A_{f,[a, b]}(\xi)=A_{f,[a, b]} \cap\{\xi\} \times \mathbb{R} . .
$$

Obviously, $A(\xi)$ consists of all real roots of the polynomial $f(\xi, x)$. Our result is given as follows.

Theorem 2. There exists a continuous mapping

$$
\gamma: t \in[0,1] \longrightarrow \gamma(t)=(\xi(t), x(t)) \in A \subset \mathbb{R}^{2},
$$

such that $\gamma(0) \in A(a)$ and $\gamma(1) \in A(b)$ are odd-multiple real roots. Moreover, let

$$
\gamma=\{\gamma(t) \mid t \in[0,1]\}, T_{p}=\{t \in[0,1] \mid \gamma(t)=P\},
$$

then for $P \in \gamma$, we have

$$
T_{p}^{\#} \leq \text { the multiplicity of } P \text {, }
$$

where $T_{P}^{\#}$ indicates the cardinality of the set $T_{P}$. Furthermore, the sets $\left\{P \in \gamma \mid T_{P}^{\#} \geq 2\right\}$ and $\gamma \cap A^{\text {even }}$ are at most countable.

Remark 3. Theorem 2 describes some information of topological structure of the set of real roots of $f$. Obviously, the continuous curve $\{\gamma(t) \mid t \in[0,1]\}$ is usually not unique. However, it is possible that $\gamma$ consists of all real roots of $f$, that is, $\{\gamma(t) \mid t \in[0,1]\}=A$. So the result in Theorem 2 is optimal. Moreover, (11) implies that

$$
\{t \in[0,1] \mid \xi(t)=\eta\}^{\#} \leq 2 d+1, \quad \forall \eta \in[a, b] .
$$

Thus, there exists an at most countable subset $I \subset[0,1]$ such that $\gamma: t \in[0,1] \backslash I \longrightarrow \gamma(t) \in A$ is an injective mapping.

The result of Theorem 2 is a global property of real roots of odd-degree real polynomials. For some local property of real roots of real polynomials, we refer to [14] and the references therein.

\section{Proof of Theorem 2}

In order to prove Theorem 2, we give some important definitions.

3.1. Definitions. Below, we first define paths of odd-multiple real roots, crossing points, basis points, and reflecting points.

Definition 4 (paths of odd-multiple real roots). Let

$$
\gamma: t \in[0,1] \longrightarrow \gamma(t)=(\xi(t), x(t)) \in A \subset \mathbb{R}^{2},
$$

be a continuous mapping with $P_{0}=\gamma(0)=\left(\xi_{0}, x_{0}\right)$ and $P_{1}=$ $\gamma(1)=\left(\xi_{1}, x_{1}\right) \cdot \gamma$ is called a path of odd-multiple real roots with endpoints $P_{0}$ and $P_{1}$ if the following hold:

(i) Let $t_{0} \in(0,1)$ and $P=\gamma\left(t_{0}\right)=\left(\xi_{*}, x_{*}\right)$, then one of the following holds true:

(1) There exists $\delta_{0}>0$ such that

$\xi(t)<\xi_{*}, \quad \forall t \in\left(t_{0}-\delta_{0}, t_{0}\right)$ and $\xi(t)>\xi_{*}, \quad \forall t \in\left(t_{0}, t_{0}+\delta_{0}\right)$,

or

$\xi(t)>\xi_{*}, \quad \forall t \in\left(t_{0}-\delta_{0}, t_{0}\right)$ and $\xi(t)<\xi_{*}, \quad \forall t \in\left(t_{0}, t_{0}+\delta_{0}\right)$

Moreover, such $t_{0}$ is unique for the point $P$ if it exists. 


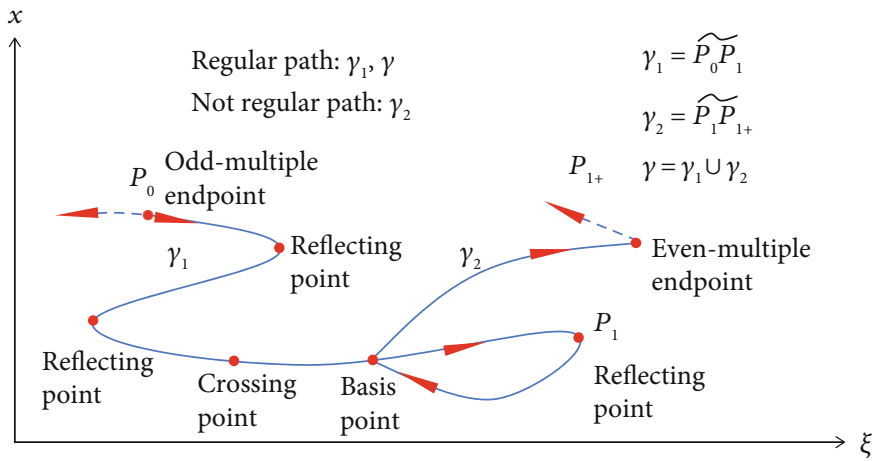

Figure 1: Path of odd-multiple real roots.

(2) There exists $\delta_{0}>0$ such that

$\xi(t)<\xi_{*}, \quad \forall 0<\left|t-t_{0}\right|<\delta_{0}$, or $\xi(t)>\xi_{*}, \quad \forall 0<\left|t-t_{0}\right|<\delta_{0}$.

(ii) Let $\tilde{T}_{p}=\{t \in(0,1) \mid \gamma(t)=P\}$. If $P \in \gamma$ is an oddmultiple real root such that there exists a unique $t_{0} \in \tilde{T}_{p}$ such that (1) holds, then such $P$ is called a crossing point at $t_{0}$. Moreover, for $t_{0}^{\prime} \in \tilde{T}_{p}$, if $t_{0}^{\prime} \neq$ $t_{0}$, the result (2) holds at $t_{0}^{\prime}$; such $P$ is called a basis point at $t_{0}^{\prime}$. If $P$ is a crossing point at $t_{0}$ and a basis point at $t_{0}^{\prime}$, we say that the basis point $P$ at $t_{0}^{\prime}$ is based on the crossing point $P$ at $t_{0}$. If $P \in \gamma$ is an oddmultiple real root such that the result (2) holds for all $t_{0} \in \tilde{T}_{p}$, then $P$ is a basis point, but not a crossing point; such $P$ is called a pure basis point

(iii) If $P \in \gamma$ is an even-multiple real root, then only the result (2) holds. An even-multiple real root on $\gamma$ is called a reflecting point

(iv) If $P_{0}=P_{1}$ is an odd-multiple real root and it is not a crossing point, then there exists $\delta>0$ such that $\{\gamma(t), 0<t<\delta\}$ and $\{\gamma(t), 1-\delta<t<1\}$ are on the same side of $\xi=\xi_{0}=\xi_{1}$

(v) If $P_{0}=P_{1}$ is an odd-multiple real root and it is a crossing point, then there exists $\delta>0$ such that $\{\gamma(t), 0<t<\delta\}$ and $\{\gamma(t), 1-\delta<t<1\}$ are on different sides of $\xi=\xi_{0}=\xi_{1}$

(vi) If $P_{0}=P_{1}$ is an even-multiple real root, then there exists $\delta>0$ such that $\{\gamma(t), 0<t<\delta\}$ and $\{\gamma(t)$, $1-\delta<t<1\}$ are on different sides of $\xi=\xi_{0}=\xi_{1}$

(vii) All basis points and reflecting points on $\gamma$ are at most countable; thus, the points on $\gamma$ are oddmultiple real roots almost everywhere

A path of odd-multiple real roots is also called a path in brief if without any confusion (see Figure 1).
By definition, an odd-multiple real root on a path can be both a crossing point and a basis point. Moreover, a path can have no pure basis point, and all odd-multiple real roots on the path can be crossing points except for its endpoints. This kind of special path is very important for the extension of paths in our problem.

Definition 5. Let $\gamma=\{\gamma(t) \mid 0 \leq t \leq 1\}$ be a path with endpoints $P_{0}=\gamma(0)$ and $P_{1}=\gamma(1)$. Let $\gamma^{\circ}=\{\gamma(t) \mid t \in(0,1)\}$, which is called the interior point of $\gamma$. If for all $P \in \gamma^{\circ} \cap$ $A^{\text {odd }}, P$ is a crossing points of $\gamma, \gamma$ is called a regular path. A regular path has no pure basis point.

Let $[a, b] \subset[0,1]$. The set $\gamma[\mathrm{a}, \mathrm{b}]=\{\gamma(t) \mid t \in[a, b]\}$ is called a segment of $\gamma$. By definition, it is easy to see that a segment of a path is still a path, but a segment of a regular path may not be regular. For $P \in \gamma, P$ may be a crossing point on one segment and a pure basis point on another (also see Figure 1).

An endpoint of a path is such a point that the path can be extended longer at this point.

Let $P_{1} \neq P_{0}$. There exists a small $\delta>0$ such that for all $0<\mathrm{t} \leq \delta, \xi(t)$ is on the same side of $\xi_{0}$. It is easy to see that we can always extend $\gamma$ to cross $P_{0}$. The same is true for $P_{1}$.

However, when the two endpoints of a path collide into one point, the path may not be extended again at the point; in this situation, the endpoints disappear. To avoid the endpoints losing their property as endpoints, we suggest the conditions (iv), (v), and (vi). More precisely, when two endpoints meet, they still keep the property of endpoints only after they collide into a pure basis point along the same side, into a crossing point along different sides, or into a reflecting point along different sides.

Usually, we can extend a path at an endpoint, but the direction of extension about the parameter $\xi$ may be restricted. For this problem, we define directions of endpoints.

Definition 6 (simple endpoints and directions of endpoints). Let

$$
\gamma: t \in[0,1] \longrightarrow \gamma(t)=(\xi(t), x(t)) \in A \subset \mathbb{R}^{2},
$$


be a path of odd-multiple real roots with endpoints $P_{0}=$ $\left(\xi_{0}, x_{0}\right)$ and $P_{1}=\left(\xi_{1}, x_{1}\right)$.

We only consider $P_{0}$ since $P_{1}$ can be considered similarly. If $P_{0}$ is neither a crossing point, nor a basis point, nor a reflecting point, we call it a simple endpoint. Moreover, if $P_{0}$ is an odd(even)-multiple real root, we call it an odd(even)multiple endpoint.

Below, we define the direction of endpoints in two cases of $P_{0} \neq P_{1}$ and $P_{0}=P_{1}$. First, note that there exists a sufficiently small $\delta_{0}>0$ such that either $\xi(t)>\xi_{0}$ for all $t \in(0$, $\left.\delta_{0}\right)$ or $\xi(t)<\xi_{0}$ for all $t \in\left(0, \delta_{0}\right)$. That means that for sufficiently small $t, \gamma(t)$ are on the same side of $\xi=\xi_{0}$.

Case $1\left(P_{0} \neq P_{1}\right)$. If $P_{0}$ is not a crossing point of the path $\gamma$ and $\gamma(t)$ are on the left side (or right side) of $\xi=\xi_{0}$ for all sufficiently small $t>0, P_{0}$ is called a right (or left) endpoint. If $P_{0}$ is a crossing point of $\gamma$ and $\gamma(t)$ are on the left side (or right side) of $\xi=\xi_{0}$ for all sufficiently small $t>0, P_{0}$ is called a left (or right) endpoint.

If $P_{0}$ is an even-multiple real root and for all sufficiently small $t>0$, and $\gamma(t)$ are on the left side (or right side) of $\xi=\xi_{0}, P_{0}$ is called a left (or right) endpoint. For $P_{1}$, we have the same statement and we omit the details.

Case $2\left(P_{0}=P_{1}\right)$. In the condition (iv) of Definition 4 , if there exists $\delta>0$ such that the set $\{\gamma(t) \mid 0<t<\delta\}$ is on the right (left) side of $\xi=\xi_{0}=\xi_{1}$, we call $P_{0}$ a left (right) endpoint and $P_{1}$ a right (left) endpoint.

In the condition (v) of Definition 4 , if the set $\{\gamma(t) \mid 0<$ $t<\delta\}$ is on the right (left) side of $\xi=\xi_{0}=\xi_{1}$, we call $P_{0}$ a right (left) endpoint and $P_{1}$ a left (right) endpoint.

In the condition (vi) of Definition 4 , if the set $\{\gamma(t) \mid 0$ $<t<\delta\}$ is on the right (left) side of $\xi=\xi_{0}=\xi_{1}$, we call $P_{0}$ a right (left) endpoint and $P_{1}$ a left (right) endpoint.

Remark 7. A left (or right) endpoint means that the path can be extended to the left (or right) of $\xi=\xi_{0}$ at the endpoint. In view of this point, in the cases of (iv)-(vi), we can define different directions of the endpoints. We choose the above definition of directions of endpoints for convenience in the extension of paths.

Definition 8 (compatibility of paths). Let $\gamma_{1}, \gamma_{2}, \cdots \gamma_{n} \subset A$ be paths of odd-multiple real roots and

$$
E=\left\{\gamma_{j}(0), \gamma_{j}(1) \mid 1 \leq j \leq n\right\}
$$

be the set of all endpoints of $\left\{\gamma_{j}\right\}$. Let $G=\gamma_{1} \cup \gamma_{2} \cup \cdots \cup \gamma_{n}$ and $\stackrel{\circ}{G}=\dot{\circ}_{1} \cup \dot{\gamma}_{2} \cup \cdots \cup \dot{\gamma}_{n}$.

If for all $P \in \stackrel{\circ}{G} \cap A^{\text {odd }}$, there exists a unique $\gamma_{j}$ such that $P$ is a crossing point of $\gamma_{j}$; moreover, if $P \in \gamma^{\circ}{ }_{k}$ with $k \neq j, P$ is a pure basis point of $\gamma_{k}$, then we say $\left\{\gamma_{j}\right\}$ are compatible (at $P$ ). If $P \in \stackrel{\circ}{G} \cap A^{\text {even }}, P$ is always a reflecting point and we also say $\left\{\gamma_{j}\right\}$ are compatible at $P$.
Let $D$ be a domain of rectangles. If for all $P \in D \cap \stackrel{\circ}{G},\left\{\gamma_{j}\right\}$ are compatible at $P$, we say $\left\{\gamma_{j}\right\}$ are compatible on $D$ or $G$ is regular on $D$.

Let $\left\{\gamma_{j}\right\}$ be compatible paths and $\gamma$ be a path of oddmultiple real roots with two endpoints $P_{0}=\gamma(0)$ and $P_{1}=$ $\gamma(1)$. If $\gamma, \gamma_{1}, \cdots \gamma_{n}$ are compatible, we say $\gamma$ is compatible with $\left\{\gamma_{j}\right\}$. Let $\widehat{\gamma}$ be a path of odd-multiple real roots with two endpoints $\widehat{P}_{0}=\widehat{\gamma}=(0)$ and $\widehat{P}_{0}=\widehat{\gamma}(1)$. Let $\widehat{P}_{0}=P_{1} \notin \mathrm{E}$. If $\gamma_{+}=\gamma \cup \widehat{\gamma}$ is a path with two endpoints $P_{0}$ and $\widehat{P}_{1}$ and it is also compatible with $\left\{\gamma_{j}\right\}$, we say $\gamma_{+}$is a compatible extension of $\gamma$ with $\left\{\gamma_{j}\right\}$ and $\widehat{\gamma}$ is the prolonged segment. Obviously, if $P_{1} \notin \stackrel{\circ}{G}$, then $P_{1}$ must be a crossing point of $\gamma_{+}$; if $P_{1} \in \stackrel{\circ}{G}$, then $P_{1}$ must be a pure basis point of $\gamma_{+}$.

Remark 9. When $\left\{\gamma, \gamma_{1}, \cdots \gamma_{n}\right\}$ are compatible, formally, we can regard $\gamma \cup \gamma_{1} \cup \cdots \cup \gamma_{n}$ as a generalized regular path, because it may not be connected and can have many endpoints. In particular, if $G=\gamma_{1} \cup \cdots \cup \gamma_{n}=\varnothing$, then $\gamma$ is a regular path.

Let $\gamma$ and $\widehat{\gamma}$ be two compatible paths. Let $P$ be an endpoint of both $\gamma$ and $\widehat{\gamma}$ with $P=\gamma(1)=\widehat{\gamma}(0)$. If $P \in A^{\text {odd }}$ and it has opposite endpoint directions for $\gamma$ and $\hat{\gamma}$, or if $P \in A^{\text {even }}$ and it has the same endpoint direction, then $\gamma_{+}=\gamma \cup \widehat{\gamma}$ is a compatible extension of $\gamma$.

Now suppose $\gamma: t \in[0,1] \longrightarrow \gamma(t) \in A$ and $\widehat{\gamma}: t \in$ $[1,2] \longrightarrow \widehat{\gamma}(t) \in A$ and $P_{*}=\gamma(1)=\widehat{\gamma}(1)$, where we use $\widehat{\gamma}(t+1)$ in place of the above $\widehat{\gamma}(t)$ given by standard definition without confusion.

Note that we can require a path to be defined on any interval $[a, b]$ instead of $[0,1]$ by rescaling the parameter of paths.

If $\gamma(1)$ and $\widehat{\gamma}(1)$ are not crossing points, we can define $\gamma_{+}=\gamma \cup \widehat{\gamma}$ as a regular path by

$$
\begin{array}{ll}
\gamma_{+}(t)=\gamma(t), & 0 \leq t \leq 1, \\
\gamma_{+}(t)=\widehat{\gamma}(t), & 1 \leq t \leq 2 .
\end{array}
$$

By definition, it is easy to see that $\gamma_{+}$is well defined.

If both $\gamma(1)$ and $\widehat{\gamma}(1)$ are crossing points and they have different directions of endpoints, then $\gamma_{+}$as defined above may not satisfy the definition of paths. The above definition may result in $\gamma_{+}(t)$ crossing itself at $P_{*}$ more than 1 time. To avoid $P_{*}$ being crossed repeatedly, we can rearrange the parameter at $P_{*}$ so that $\gamma_{+}=\gamma \cup \widehat{\gamma}$ can be defined as a regular path, but here, we omit the details.

Compatibility of paths is very important for extension of paths. It makes the extension of a path to not overlap any small segment of existing paths so that the extension cannot have any circulation.

When we extend a regular path, we require the path to keep the compatibility with the other existing paths. So at the first time when the path meets a new odd-multiple real root, we always let it go through the odd-multiple real root such that it becomes a crossing point of the path. When the path meets itself or some existing path at a crossing point, 
it must reflect at this point, and then it becomes a basis point. That means a new odd-multiple real root usually first becomes a crossing point and then a basis point. When the path meets an even-multiple real root, it is always extended by reflecting at this point.

We usually start a compatible extension of a regular path with a simple endpoint and require the prolonged segment to end also at a simple endpoint, so that we can easily see the extension to be compatible.

Definition 10 (closed paths). Let $\gamma$ be a path of odd-multiple real roots with endpoints $P_{0}$ and $P_{1}$. We call $\gamma$ a closed path of odd-multiple real roots, if $P_{0}=P_{1}$ with the following holding true:

(1) If $P_{0}$ is a crossing point and there exists a sufficiently small $\delta_{0}>0$ such that $\left\{\gamma(t) \mid t \in\left(0, \delta_{0}\right)\right\}$ and $\{\gamma(t) \mid$ $\left.t \in\left(1-\delta_{0}, 1\right)\right\}$ are on the same side of $\xi=\xi_{0}$, then $P_{0}$ is a crossing point of the closed path

(2) If $P_{0}$ is not a crossing point and there exists a sufficiently small $\delta_{0}>0$ such that $\left\{\gamma(t) \mid t \in\left(0, \delta_{0}\right)\right\}$ and $\left\{\gamma(t) \mid t \in\left(1-\delta_{0}, 1\right)\right\}$ are on different sides of $\xi=\xi_{0}$, then $P_{0}$ is a crossing point of the closed path

(3) If $P_{0}$ is not a crossing point and there exists a sufficiently small $\delta_{0}>0$ such that $\left\{\gamma(t) \mid t \in\left(0, \delta_{0}\right)\right\}$ and $\left\{\gamma(t) \mid t \in\left(1-\delta_{0}, 1\right)\right\}$ are on the same side of $\xi=\xi_{0}$, then $P_{0}$ is a pure basis point of the closed path

(4) If $P_{0}$ is an even-multiple real root and there exists a sufficiently small $\delta_{0}>0$ such that $\left\{\gamma(t) \mid t \in\left(0, \delta_{0}\right)\right\}$ and $\left\{\gamma(t) \mid t \in\left(1-\delta_{0}, 1\right)\right\}$ are on the same side of $\xi=\xi_{0}$, then $P_{0}$ is a reflecting point of the closed path

When $\gamma$ is a closed path, we can regard $\gamma$ as a continuous mapping: $\mathbb{R} / \mathbb{Z}=T \longrightarrow A$, which is from the unit circle $T$ to A. $P_{0}=P_{1}$ can be a crossing point or a pure basis point of the closed path (see Figure 2).

If a closed path $\gamma: t \in T=\mathbb{R} / \mathbb{Z} \longrightarrow \gamma(t) \in A \subset \mathbb{R}^{2}$ has no pure basis point, we call $\gamma$ a closed regular path; see Figure 3.

A closed regular path is a circulation and it cannot be extended longer.

3.2. Idea of Construction of Paths. Below, we give a more detailed description of the construction of paths.

Let $f(\xi, x)$ be a polynomial of degree $2 d+1$ as in (1) and

$$
\begin{aligned}
A_{2 d+1}= & \{(\xi, x) \mid x \text { is a }(2 d+1) \text {-multiple real root of } f(\xi, x), \\
& \xi \in[a, b]\}, \\
B_{2 d+1}= & \left\{\xi \mid(\xi, x) \in A_{2 d+1}\right\} .
\end{aligned}
$$

Obviously, $f_{x}^{(2 d)}(\xi, x)=0$ determines a simple continuous curve

$$
l: x=-\frac{1}{2 d+1} c_{1}(\xi), \quad \xi \in[a, b]
$$

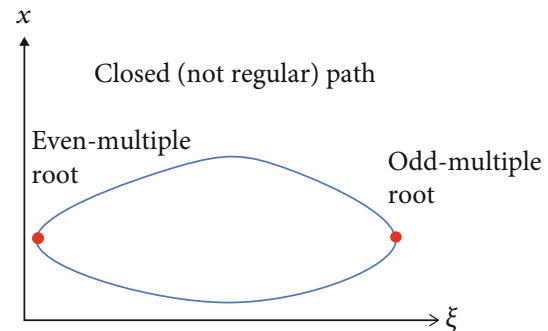

FIgURE 2: Closed path.

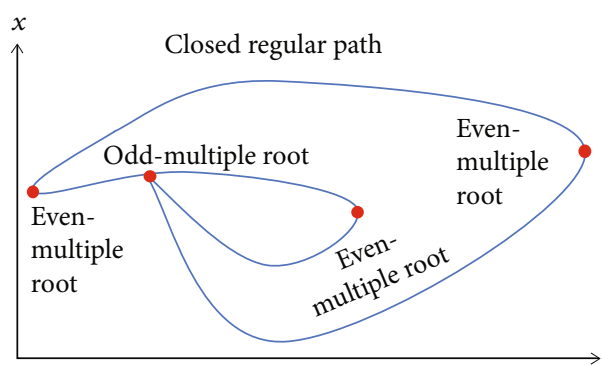

FIgURE 3: Closed regular path.

Moreover, $A_{2 \mathrm{~d}+1} \subset \ell$. Since $\mathrm{L}=(a, b) \backslash B_{2 \mathrm{~d}+1}$ is an open set of $\mathrm{R}$, then $\mathrm{L}=\cup_{\mathrm{n} \geq 1} I_{n}$, where $\mathrm{I}_{\mathrm{n}}=\left(a_{n}, b_{n}\right) \subset(a, b)$, which are disjointed each other.

Let $\ell_{n}$ be the segment of $\ell$ corresponding to $\ell_{n}$. We want to find a path $\gamma_{n}$ in $A_{f,\left[a_{n}, b_{n}\right]}$ in place of $\ell_{n}$.

Moreover, we also find paths $\gamma_{ \pm}$in $A_{f,\left[a_{ \pm}, b_{ \pm}\right]}$for $\xi \in\left[a_{-}, b_{-}\right]$ and $\xi \in\left[a_{+}, b_{+}\right]$, respectively, where $a_{-}=a, b_{-}=\min \{\xi \in$ $\left.B_{2 d+1}\right\}$ and $a_{+}=\max \left\{\xi \in B_{2 d+1}\right\}, b_{+}=b$, if $a$ or $b$ does not belong to $B_{2 d+1}$. Let $\ell_{ \pm}$be the two segments of $l$ on $\left(a_{-}, b_{-}\right)$ and $\left(a_{+}, b_{+}\right)$. Then, if we replace $l_{n}$ and $\ell_{ \pm}$in $l$ with $\gamma_{n}$ and $\gamma_{ \pm}$, respectively, we obtain a path of odd-multiple real roots for $\xi$ on $[a, b]$.

The most important is to construct a path $\gamma_{n}$ on $\left[a_{n}, b_{n}\right]$. Then, the path $\gamma$ is obtained by grafting the simple continuous curve $l$ with countable paths $\left\{\gamma_{n}\right\}$ and $\ell_{+}$, where $\gamma_{n}$ and $\ell_{ \pm}$are composed of at most countable paths of polynomials of lower degree, maybe in a little complicated way. Moreover, each $\gamma_{n}$ and $\ell_{ \pm}$include at most countable reflecting points and basis points.

To consider $\gamma_{n}$ on $\left[a_{n}, b_{n}\right]$, for shorting of notation, we drop the subscripts $n$. Then, suppose $f(a, x)$ and $f(b, x)$ have $(2 d+1)$-multiple real roots and $f(\xi, x)$ has no $(2 d+1)$ -multiple real root for $\xi \in(a, b)$. Now we explain how to construct a path $\gamma$ on $[a, b]$.

The construction of $\gamma$ is based on extension of paths and induction for the degree of polynomials.

First note that an odd-multiple real root is never isolated and usually it breaks into some lower-multiple real roots about the parameter. However, an even-multiple real root can be isolated, near to which there may be no other real roots. But, if there exists an odd-multiple real root near an even-multiple real root, there must be another odd-multiple real root near the even-multiple real root, that is, oddmultiple real roots always appear in pairs near an even- 
multiple real root. Thus, when a path arrives at an evenmultiple real root, it can always reflect into some oddmultiple real roots at the even-multiple real root. So we define a path to be such a continuous curve that it is allowed to go through an odd-multiple real root and reflect when it meets an even-multiple real root.

A path of odd-multiple real roots can be a little complicated near to a real root of higher-multiplicity; it can zigzag along the direction of the $x$-axes and have several recurrences at some parameters to approximate the real root of higher multiplicity. However, the times of the recurrence are no more than the multiplicity of the real root.

Now, we first construct a regular path to join to $P_{b}$. Let $a<\xi_{0}<b$. Now, take an odd-multiple real root on $D\left(\xi_{0}\right)$. By induction assumption, there exists a local regular path across the odd-multiple real root. Then, we extend the local regular path to the right. Since $f(\xi, x)$ has no $(2 d+1)$-multiple real root for $\xi \in(a, b)$, the real roots near any an oddmultiple real root can be all the real roots of a real polynomial of lower degree, and so we can extend it locally longer by induction assumption. We can extend the path until it arrives at $P_{b}$ or $\xi=\xi_{0}$. In the first situation, we have a desired path; otherwise, the path is extended to arrive at a new oddmultiple real root on $D\left(\xi_{0}\right)$. By induction assumption, we can extend the path across $\xi=\xi_{0}$ to the left a little and then have a regular path with two endpoints on the left of $D\left(\xi_{0}\right)$.

Then, take another odd-multiple real root on $D\left(\xi_{0}\right)$ and do the same way. If we cannot have a desired path, we have another compatible path with the previous path with two endpoints on the left of $D\left(\xi_{0}\right)$. In this way, after at most $d$ steps, only one odd-multiple real root is left on $D\left(\xi_{0}\right)$. Take a path across this odd-multiple real root and extend it to the right, since all the odd-multiple real roots on $D\left(\xi_{0}\right)$ are occupied by the previous compatible paths and the path cannot go back to $D\left(\xi_{0}\right)$. Finally, it must go to $P_{b}$, and then we obtain a regular path joining to $P_{b}$.

Then, we extend the path joining to $P_{b}$ to the left. If it is not extended to $P_{a}$, it must go back to $P_{b}$ to form a closed path with $P_{b}$ as a pure basis point. Then, take an oddmultiple real root on $D\left(\xi_{0}\right)$ and do the same way as above to obtain a compatible path joining to $P_{b}$. In the same way, we extend the compatible path joining to $P_{b}$ to the left. If we cannot have a desired path, we must have one more compatible closed path with $P_{b}$ as a pure basis point. But we cannot have more than $d$ number of these compatible closed paths, so after at most $d$ iterations, the extension of the compatible path cannot go back to $P_{b}$; it has to go to $P_{a}$. Thus, the extension of the compatible path joins with the previous several compatible closed paths to form a regular path $\gamma$ joining to $P_{a}$ and $P_{b}$.

We should also note that if two $2 d$-multiplicity real roots are sufficiently close, the two corresponding simple real roots to the two $2 d$-multiplicity real roots can be connected by a saturation path of simple real roots. So a path cannot meet two $2 d$-multiplicity real roots which are very close; in other words, all $2 d$-multiplicity real roots on a path are discrete. Thus, the path $\gamma$ can include at most countable $2 d$-multiple real roots. Moreover, $\gamma$ can only have $P_{a}$ or $P_{b}$ as $(2 d+1)$-multiple basis points. This fact is very important for a path to contain at most countable basis points and reflecting points.

3.3. Lemmas. Below, we prove some lemmas which are required in the construction of paths.

We first note that in Definition 4, the conditions (1) of (i) and (vii) are very important; they guarantee that any small segment of a path cannot be overlapped one more times by the path itself. Moreover, a path cannot recur too many times about a fixed parameter.

Lemma 11. Let $\{\gamma(t)=(\xi(t), x(t)) \mid t \in[0,1]\}$ be a path of real roots. For any two intervals $I=\left(t_{1}, t_{2}\right) \subset(0,1)$ and $I^{\prime}=$ $\left(t_{1}^{\prime}, t_{2}^{\prime}\right) \subset(0,1)$, if $I \cap I^{\prime}=\phi$, then the set $\gamma_{I} \cap \gamma_{I^{\prime}}$ is at most countable. Moreover, $T_{\xi_{0}}^{\#} \leq 2 d+1$, where $T_{\xi_{0}}=\{t \in[0,1] \mid$ $\left.\xi(t)=\xi_{0}\right\}$.

Proof. If $\gamma_{I} \cap \gamma_{I^{\prime}}$ is not countable, by the condition (vii) of the definition of paths, there exists a point $P \in\{\gamma(t) \mid t \in I\} \cap$ $\left\{\gamma(t) \mid t \in I^{\prime}\right\}$ such that $P$ is neither a basis point nor an even-multiple real root, then $P$ is a pure crossing point. Again, there exist $t_{0} \in I$ and $t_{0}^{\prime} \in I^{\prime}$ such that $P=\gamma\left(t_{0}\right)=\gamma\left(t_{0}^{\prime}\right)$, but $t_{0} \neq t_{0}^{\prime}$, which contradicts with the fact that $P$ can be crossed only once.

If there are several segments of the path crossing the parameter $\xi_{0}$, the condition (vii) implies that there exists $\xi_{n} \longrightarrow \xi_{0}$ such that each segment crossing $\xi=\xi_{0}$ does not meet the other segments on the line $\xi=\xi_{n}$. Otherwise, if for all $\left|\eta-\xi_{0}\right|<\delta_{0}$, at least two segments have an intersecting point $P_{\eta}$ on $\xi=\eta$, these segments can intersect on $\left\{P_{\eta}|| \eta-\xi_{0} \mid \leq \delta_{0}\right\}$, which has the cardinal number of the continuum and contradicts with the condition (vii).

Since the number of segments crossing the line $\xi=\xi_{0}$ is no more than that of points of these segments on $\xi=\xi_{0}$, which is no more than $2 d+1$, so the path oscillates for the parameter around $\xi_{0}$ no more than $2 d+1$ times, which implies $T_{\xi_{0}}^{\#} \leq 2 d+1$.

This lemma means that a path cannot intersect itself everywhere on a nonempty segment, which guarantees a path to recur to a fixed parameter $\xi_{0}$ no more than $2 d+1$ times. Moreover, an $m$-multiple real root can be used by a path at most $m$-times. If the multiplicity is considered and an $m$ multiple real root is regarded as $m$ different real roots, then a compatible extension can be thought to consist of new real roots and a path can be thought to consist of different real roots.

For the construction of paths, we consider how a path can intersect with a line $\xi=\xi_{0}$. For this purpose, we have the following lemma.

Lemma 12. Let $\gamma$ be a path of odd-multiple real roots with two endpoints $P_{1}=\left(\xi_{1}, x_{1}\right)$ and $P_{2}=\left(\xi_{2}, x_{2}\right)$ with $\xi_{1}<\xi_{2}$. Then, the following hold true:

(1) If $\xi_{1}<\xi_{0}<\xi_{2}$, then $\gamma \cap\left\{\xi_{0}\right\} \times \mathbb{R}$ has an odd number of crossing points 
(2) If $\xi_{0}<\xi_{1}$ or $\xi_{0}>\xi_{2}$, then $\gamma \cap\left\{\xi_{0}\right\} \times \mathbb{R}$ has an even number of crossing points.

Proof.

(1) By definition, we can move $P_{1}$ continuously to $P_{2}$ along the path $\gamma$, and in the process, we go through odd-multiple real roots only one time.

Without loss of generality, suppose $P_{1}$ and $P_{2}$ are left and right endpoints, respectively. Then, the path starts with $P_{1}$ to go to the right. Since $\xi_{0} \in\left(\xi_{1}, \xi_{2}\right)$, it must intersect with $\left\{\xi_{0}\right\} \times \mathbb{R}$ at some odd-multiple real root $Q_{1}$. If it goes back across $\left\{\xi_{0}\right\} \times \mathbb{R}$ at some odd-multiple real root $Q_{2}$, then $Q_{1} \neq Q_{2}$ since they can only be crossed only once. Then, it must cross $\left\{\xi_{0}\right\} \times \mathbb{R}$ at another odd-multiple real root $Q_{3}$; otherwise, it cannot arrive to $P_{2}$. In this way, it is easy to see that the path must cross $\left\{\xi_{0}\right\} \times \mathbb{R}$ an odd number of times.

(2) This case can be considered similarly and we omit the detail

Let

$$
D=\{(\xi, x) \mid a \leq \xi \leq b, c<x<d\},
$$

be a rectangle domain of the $(\xi, x)$-plane. Let $D(\xi)=\{\xi\} \times$ $(c, d)$ be a section of $D$ at $\xi$.

Let the boundary

$$
\partial D=l_{x}^{-} \cup l_{x}^{+} \cup l_{\xi}^{-} \cup l_{\xi}^{+},
$$

where

$$
l_{x}^{-}=[a, b] \times\{c\}, l_{x}^{+}=[a, b] \times\{d\}, l_{\xi}^{-}=D(a), l_{\xi}^{+}=D(b) .
$$

Obviously, the rectangle $D$ is open at the up-down sides $l_{x}^{ \pm}$and closed at the left-right sides $l_{\xi}^{ \pm}$. Let $D^{\circ}=(a, b) \times(c, d)$ be the interior of $D$.

Lemma 13. Let $\gamma$ be a path of odd-multiple real roots with endpoints $P_{1}$ and $P_{2}$ and $\gamma \cap l_{x}^{ \pm}=\phi$. Suppose $P_{1}, P_{2} \notin D$. Then, the number of crossing points in $\gamma \cap D(\xi)$ has the same odevity for all $\xi \in[a, b]$. In particular, if $\gamma$ does not go through $D$, then the number of crossing points in $\gamma \cap D(\xi)$ is even; if $\gamma$ goes through $D$, then the number of crossing points in $\gamma \cap D(\xi)$ is odd.

Proof. By definition of paths, it follows easily and we omit the details.

By the implicit function theorem, for a simple real root $P_{0}$, there exists a path crossing $P_{0}$, which only includes simple real roots. Below, we consider the maximal one among these paths.
Lemma 14. Suppose that both $f(a, x)$ and $f(b, x)$ have a $(2 d+1)$-multiple real root. Fix $\xi_{0} \in(a, b)$. Let

$$
f\left(\xi_{0}, x_{0}\right)=0, f_{x}\left(\xi_{0}, x_{0}\right) \neq 0 .
$$

There exists a unique continuous function $x=x(\xi), \xi \in$ $\left(a_{0}, b_{0}\right) \subset(a, b)$ such that

$$
x_{0}=x\left(\xi_{0}\right), f(\xi, x(\xi))=0, f_{x}(\xi, x(\xi)) \neq 0, \quad \forall \xi \in\left(a_{0}, b_{0}\right) .
$$

Moreover,

$$
\begin{aligned}
& f\left(a_{0}, x_{a_{0}}\right)=f_{x}\left(a_{0}, x_{a_{0}}\right)=f\left(b_{0}, x_{b_{0}}\right)=f_{x}\left(b_{0}, x_{b_{0}}\right)=0, \\
& f\left(a_{0}, x_{a_{0}}\right)=f_{x}\left(a_{0}, x_{a_{0}}\right)=f\left(b_{0}, x_{b_{0}}\right)=f_{x}\left(b_{0}, x_{b_{0}}\right)=0 .
\end{aligned}
$$

The continuous curve $\gamma:\left\{(\xi, x(\xi)) \mid \xi \in\left(a_{0}, b_{0}\right)\right\}$ is called a maximal (or saturation) path of the simple root $P_{0}=\left(\xi_{0}, x_{0}\right)$.

Proof. By the implicit function theorem, there exists $\delta_{0}>0$ and a unique implicit function $x=x(\xi), \xi \in\left(\xi_{0}-\delta, \xi_{0}+\delta\right)$ such that

$$
x\left(\xi_{0}\right)=x_{0}, f(\xi, x(\xi))=0, f_{x}(\xi, x(\xi)) \neq 0, \xi \in\left(\xi_{0}-\delta, \xi_{0}+\delta\right) .
$$

Below, we extend the continuous function $x=x(\xi)$ from $\left(\xi_{0}-\delta, \xi_{0}+\delta\right)$ to an interval as big as possible.

Let

$\mathscr{J}=\{[\bar{a}, \bar{b}] \mid$ there exists a unique function $x=x(\xi), \xi \in[\bar{a}, \bar{b}]$, such that $x\left(\xi_{0}\right)=x_{0}, f(\xi, x(\xi))=0, f_{x}(\xi, x(\xi)) \neq 0$,

$$
\xi \in[\bar{a}, \bar{b}]\} \text {. }
$$

Obviously, $\xi_{0} \in(\bar{a}, \bar{b})$. Let

$$
a_{0}=\inf _{[\bar{a}, \bar{b}] \in \mathcal{J}}=\bar{a}, b_{0}=\sup _{[\bar{a}, \bar{b}] \in \mathscr{\mathcal { J }}} \bar{b} .
$$

For $\xi \in\left(a_{0}, b_{0}\right)$, we define $x_{*}(\xi)=x(\xi), \xi \in(\bar{a}, \bar{b})$.

We prove that $x_{*}(\xi)$ is well defined on $\left(a_{0}, b_{0}\right)$. In fact, $x(\xi), \xi \in[\bar{a}, \bar{b}]$ is determined uniquely by $\left(\xi_{0}, x_{0}\right)$. It is easy to see that there exists $\alpha>0$ such that

$$
\left|f_{x}(\xi, x(\xi))\right| \geq \alpha>0, \quad \xi \in[\bar{a}, \bar{b}] .
$$

By the implicit function theorem, there exists $\delta>0$ such that for all $\eta \in(\bar{a}, \bar{b})$, on the interval $[\eta-\delta, \eta+\delta]$, there uniquely determines an implicit function $x(\xi)$ such that

$$
f(\xi, x(\xi))=0, \xi \in[\eta-\delta, \eta+\delta] .
$$


This implies that $x=x(\xi)$ can be obtained uniquely by extension of $x=x(\xi)$ from $\xi \in\left(\xi_{0}-\delta, \xi_{0}+\delta\right)$ to $[\bar{a}, \bar{b}]$.

Below, we prove $\lim _{\xi \rightarrow a_{0}^{+}} x_{*}(\xi)=x_{a_{0}}$. Note that $f\left(\xi, x_{*}(\xi)\right)=$ $0, f_{x}\left(\xi, x_{*}(\xi)\right) \neq 0$. First, we prove the existence of the limit. If not so, there exist $\xi_{j}^{(1)} \longrightarrow a_{0}^{+}$and $\xi_{j}^{(2)} \longrightarrow a_{0}^{+}$such that $x_{*}$ $\left(\xi_{j}^{(1)}\right) \longrightarrow x_{1}$ and $x_{*}\left(\xi_{j}^{(2)}\right) \longrightarrow x_{2}$ with $x_{1}<\mathrm{x}_{2}$.

Take $x_{1}^{*}, x_{2}^{*}$ such that $x_{1}<x_{1}^{*}<x_{2}^{*}<x_{2}$. Without loss of generality, suppose

$$
x_{*}\left(\xi_{j}^{(1)}\right) \leq x_{1}^{*}, x_{*}\left(\xi_{j}^{(2)}\right) \geq x_{2}^{*}
$$

Since $x_{*}(\xi)$ is continuous, for all $x^{*}$ such that $x_{1}^{*} \leq x^{*} \leq x_{2}^{*}$, there exists $\xi_{j}^{*}$ between $\xi_{j}^{(1)}$ and $\xi_{j}^{(2)}$ such that $x_{*}\left(\xi_{j}^{*}\right)=x^{*}$. Also note $\xi_{j}^{*} \longrightarrow a_{0}^{+}$. By $f\left(\xi_{j}{ }^{*}, x_{*}\left(\xi_{j}{ }^{*}\right)\right)=0$, it follows that

$$
f\left(a_{0}, x^{*}\right)=0, \quad \forall x^{*} \in\left[x_{1}^{*}, x_{2}^{*}\right] .
$$

Thus, all $x^{*} \in\left[x_{1}^{*}, x_{2}^{*}\right]$ are real roots of $f\left(a_{0}, x\right)$. Since $f\left(a_{0}, x\right)=0$ has at most $2 d+1$ real roots, this is a contradiction, which implies the existence of $\lim _{\xi \rightarrow a_{0}^{+}} x_{*}(\xi)$.

Similarly, $\lim _{\xi \rightarrow b_{0}^{-}} x_{*}(\xi)$ also exists.

Obviously, we have $f\left(a_{0}, x_{*}\left(a_{0}\right)\right)=0$. If $f_{x}\left(a_{0}, x_{*}\left(a_{0}\right)\right) \neq$ 0 , we can extend $x_{*}(\xi)$ at $\xi=a_{0}$ to a small neighbourhood of $a_{0}$, which contradicts with the definition of $a_{0}$.

The case of $\xi=b_{0}$ can be discussed similarly.

Remark 15. Let $\left|f_{x}\left(\xi_{0}, x_{0}\right)\right| \geq \alpha$ and $f(\xi, x)$ is continuous on $D=[a, b] \times[-1,1]$ and $\left|f_{x}(\xi, x)\right| \leq M,\left|f_{x x}(\xi, x)\right| \leq M, \forall(\xi, x)$ $\in[a, b] \times[-1,1]$. By the proof of the implicit theorem, it is easy to see that there exists constant $\delta>0$, which is independent of $\left(\xi_{0}, x_{0}\right)$ and only depends on $\alpha, M$, and the property of uniform continuity of $f$ on $D$, such that $x(\xi)$, the implicit function across $\left(\xi_{0}, x_{0}\right)$, is defined for $\xi \in\left(\xi_{0}-\delta, \xi_{0}+\delta\right) \subset$ $\left(a_{0}, b_{0}\right)$. In general, the bigger the derivative $\left|f_{x}\left(\xi_{0}, x_{0}\right)\right|$, the longer the interval $\left(a_{0}, b_{0}\right)$. Moreover, we have that $\delta \longrightarrow 0$ as $f_{x}\left(\xi_{0}, x_{0}\right) \longrightarrow 0$.

3.4. 3-Degree Polynomials. The proof of our result is strongly based on induction; for this purpose, we first consider 3degree polynomials. Let

$$
f(\xi, x)=x^{3}+c_{1}(\xi) x^{2}+c_{2}(\xi) x+c_{3}(\xi),
$$

where $c_{j}(j=1,2,3)$ are continuous on $[a, b]$.

Let

$A_{m}=\{(\xi, x) \mid(\xi, x)$ is an $m$-multiple real root of $f, \xi \in[a, b]\}$,

$$
B_{m}=\left\{\xi \mid(\xi, x) \in A_{m}\right\}, \quad m=1,2,3 .
$$

Let $A=A_{1} \cup A_{2} \cup A_{3}$, which is the set of all real roots of $f$. For $\xi \in B_{2}$, let $P_{\xi}^{1}=\left(\xi, x_{\xi}^{1}\right)$ and $P_{\xi}^{2}=\left(\xi, x_{\xi}^{2}\right)$ be, respectively, the simple real root and the 2-multiple real root of $f(\xi, x)$. For $\xi \in A_{3}$, let $P_{\xi}=(\xi, x)$ be the unique 3multiple real root of $f(\xi, x)$.

Lemma 16. Assume $A_{3}=\varnothing$ and $B_{2}=\{a, b\}$. Then, there exists a regular path in $A$ joining with $P_{a}^{1}$ and $P_{b}^{1}$.

Proof. Denote by $\ell_{P^{1}}$ the maximal path across the simple root $P_{a}^{1}$. By Lemma 14, it must end at some multiple real root. Since there is no multiple real root between $\xi=a$ and $\xi=b$, on the right side, $\ell_{P_{a}^{1}}$ goes through $P_{b}^{1}$ or ends at $P_{b}^{2}=\left(b, x_{b}^{2}\right)$.

If $\ell_{P_{a}^{1}}$ does not reach $P_{b}^{1}$, it must go to $P_{b}^{2}$. Note that $P_{b}^{2}$ is a 2-multiple real root, $f$ has a simple real $\operatorname{root}\left(\xi_{0}, x_{0}\right)$ such that $a<\xi_{0}<\mathrm{b}$ and $\left(\xi_{0}, x_{0}\right) \notin \ell_{P_{a}^{1}}$; moreover, $\left(\xi_{0}, x_{0}\right)$ is sufficiently close to $P_{b}^{2}$. Then, the maximal path across $\left(\xi_{0}, x_{0}\right)$ ends at

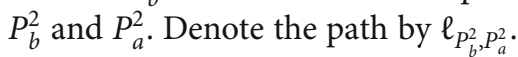

On the other hand, since the maximal path $\ell_{P_{b}^{1}}$ across $P_{b}^{1}$ cannot go to $P_{a}^{1}$, it must end at $P_{a}^{2}$ on the left. Thus, $\ell_{P_{a}^{1}} \cup$ $\ell_{P_{b}^{2}, P_{a}^{2}} \cup \ell_{P_{b}^{1}}$ forms a path joining with $P_{a}^{1}$ and $P_{b}^{1}$.

If $\ell_{P_{a}^{1}}$ goes across $P_{b}^{1}$, then it is just a path joining with $P_{a}^{1}$ and $P_{b}^{1}$.

Obviously, the path has no basis point and so it is a regular path. Thus, the lemma is proven.

Lemma 17. Assume that $A_{3}=\varnothing$ and $a, b \in B_{2}$. Then, there exists a regular path between $\xi=a$ and $\xi=b$, which joins with $P_{a}^{1}$ and $P_{b}^{1}$. Moreover, it includes at most finitely many 2multiple real roots.

Proof. Obviously, if $B_{2}=\{a, b\}$, it is just the case in Lemma 16. Assume $B_{2} \backslash\{a, b\} \neq \varnothing$.

Let $d_{0}=\inf _{\xi \in B_{2}}\left|x_{\xi}^{2}-x_{\xi}^{1}\right|$.

We first claim that there exists $\xi_{0} \in B_{2}$ such that $d_{0}=1$ $x_{\xi_{0}}^{2}-x_{\xi_{0}}^{1} \mid>0$.

In fact, we first have $d_{0}>0$. If not so, there exist $\xi_{n} \in B_{2}$ such that $\left|x_{\xi_{n}}^{1}-x_{\xi_{n}}^{1}\right| \longrightarrow 0$. Without loss of generality, assume $x_{\xi_{n}}^{2} \longrightarrow x_{0}, x_{\xi_{n}}^{1} \longrightarrow x_{0}, \xi_{n} \longrightarrow \xi_{0}$. Then, $\left(\xi_{0}, x_{0}\right)$ is a 3multiple real root of $f$, which is a contradiction.

Now take $\xi_{n} \in B_{2}$ such that $\left|x_{\xi_{n}}^{2}-x_{\xi_{n}}^{1}\right| \longrightarrow d_{0}$. Since $B_{2}$ is closed, there exists a subsequence $\xi_{n_{k}} \longrightarrow \xi_{0} \in B_{2}$. Without loss of generality, assume $\xi_{n} \longrightarrow \xi_{0}$. Since $\left|x_{\xi_{0}}^{2}-x_{\xi_{0}}^{1}\right| \geq d_{0}$, the maximal path across $P_{\xi_{0}}^{1}=\left(\xi_{0}, x_{\xi_{0}}^{1}\right), l_{P_{\xi_{0}}^{1}}$, exists for $\mid \xi-\xi_{0}$ $\mid \leq \delta_{0}$, where $\delta_{0}$ is a small constant. Let $z_{1}(\xi), z_{2}(\xi), z_{3}(\xi)$ be the three continuous bifurcations of roots of $f$. Then, $l_{P_{\xi_{0}}^{1}}$ must be on a continuous bifurcation. Without loss of generality, assume

$$
l_{P_{\xi_{0}}^{1}} \subset\left\{\left(\xi, z_{1}(\xi)\right) \mid \xi \in[a, b]\right\}
$$

Then, $z_{1}\left(\xi_{0}\right)=x_{\xi_{0}}^{1}$. It follows that

$$
x_{\xi_{n}}^{1}=z^{1}\left(\xi_{n}\right) \longrightarrow z_{1}\left(\xi_{0}\right)=x_{\xi_{0}}^{1} .
$$


Noting that $z_{2}\left(\xi_{0}\right)=z_{3}\left(\xi_{0}\right)=x_{\xi_{0}}^{2}$ and

$$
x_{\xi_{n}}^{2}=z_{2}\left(\xi_{n}\right)=z_{3}\left(\xi_{n}\right) \longrightarrow z_{2}\left(\xi_{0}\right)=z_{3}\left(\xi_{0}\right)=x_{\xi_{0}}^{2},
$$

we have $\left|x_{\xi_{n}}^{2}-x_{\xi_{n}}^{1}\right| \longrightarrow\left|x_{\xi_{0}}^{2}-x_{\xi_{0}}^{1}\right|$ and then $\left|x_{\xi_{0}}^{2}-x_{\xi_{0}}^{1}\right|=d_{0}$.

Moreover, it follows easily that

$$
\left|f_{x}\left(\xi, x_{\xi}^{1}\right)\right| \geq d_{0}^{2}, \quad \forall \xi \in B_{2} .
$$

By Lemma 14 and Remark 15, there exists $\delta>0$, which is independent of $\xi \in B_{2}$, such that for any $\eta \in B_{2}$, a maximal path $\ell_{\left(\eta, x_{\eta}^{1}\right)}$ is defined well for $|\xi-\eta| \leq \delta$. More precisely, $(\eta-\delta, \eta+\delta)$ is included in the definition interval of the maximal path.

Let $a=\xi_{0}<\xi_{1}<\cdots \xi_{n}=b$ such that $\left|\xi_{j}-\xi_{j-1}\right| \leq \delta, j=1$, $2 \cdots n$.

If $\left[\xi_{j-1}, \xi_{j}\right] \cap B_{2} \neq \varnothing$, let

$a_{j}=\min \left\{\xi \mid \xi \in B_{2} \cap\left[\xi_{j-1}, \xi_{j}\right]\right\}, b_{j}=\max \left\{\xi \mid \in B_{2} \cap\left[\xi_{j-1}, \xi_{j}\right]\right\}$.

Obviously, $\xi_{j-1} \leq a_{j} \leq b_{j} \leq \xi_{j}$ and $b_{j}-a_{j} \leq \delta$. By Lemma 14 and using the same notation as in Lemma 16, the maximal path $\ell_{P_{a_{j}}^{1}}$ joins with $P_{a_{j}}^{1}$ and $P_{b_{j}}^{1}$. Denote by $\bar{\ell}_{j}=\ell_{P_{a j}^{1}, P_{b j}^{1}}$ the segment of $\ell_{P_{a_{j}}^{1}}$ joining with $P_{a_{j}}^{1}$ and $P_{b_{j}}^{1}$. If $a_{j}=b_{j}$, we let $l_{P_{a_{j}}^{1}}=P_{a_{j}}^{1}$. Obviously, there is no 2-multiple real root on the path $\ell_{P_{a_{j}}^{1}}$.

If $\left[\xi_{j-1}, \xi_{j}\right] \cap B_{2}=\varnothing$, we have no such path.

All these paths can be arranged as $\left\{l_{p_{a_{j_{k}}}^{1}}\right\}_{k=1}^{m}$ with $m \leq n$ and

$$
a=a_{j_{1}} \leq b_{j_{1}} \leq \cdots a_{j_{k}} \leq b_{j_{k}} \leq a_{j_{k+1}} \leq b_{j_{k+1}} \leq a_{j_{m}} \leq b_{j_{m}}=b .
$$

In particular, if $\xi \in\left(b_{j_{k}}, \mathrm{a}_{\mathrm{j}_{\mathrm{k}+1}}\right), f(\xi, x)$ has no 2-multiple real root. By Lemma 16, there exists a regular path

$$
\tilde{\ell}_{j_{k}}=\ell_{P_{b_{j_{k}}}^{1}, P_{a_{k+1}}^{1}},
$$

such that $\tilde{\ell}_{j_{k}}$ joins with $P_{b_{j_{k}}}^{1}$ and $P_{a_{j_{k+1}}}^{1}$.

If $a_{j_{k+1}}-b_{j_{k}}<\delta$, then $\tilde{\ell}_{j_{k}}$ is a segment of the maximal path of the simple real root $P_{b_{j_{k}}}$, on which there is no 2-multiple real root. Only if $a_{j_{k+1}}-b_{j_{k}}<\delta$ can the 2-multiple real roots $P_{b_{j_{k}}}^{2}$ and $P_{a_{j_{k+1}}}^{2}$ be on $\tilde{\ell}_{j_{k}}, k=1,2, \cdots m-1$.

It is easy to see that all the paths

$$
\bar{\ell}_{j_{1}}, \tilde{\ell}_{j_{1}}, \cdots \bar{\ell}_{j_{k}}, \tilde{\ell}_{j_{k}} \cdots \bar{\ell}_{j_{m}}, \tilde{\ell}_{j_{m}}
$$

can be continuously connected to form a regular path joining with $P_{a}^{1}$ and $P_{b}^{1}$. Moreover, it includes at most these 2multiple real roots $\left\{P_{b_{j_{k}}}^{2}, P_{a_{j_{k+1}}}^{2}\right\}_{k=1}^{m}$.

By rescaling the parameter of the paths, we can define the path as a continuous mapping from $[0,1]$ to $A$, but we omit the details and refer to the proof of Lemma 26 below, where a more general case is considered.

Lemma 18. Assume $B_{3}=\{a, b\}$. Then, there exists a regular path $\ell$ in $A$ joining with $P_{a}$ and $P_{b}$; moreover, $\ell$ includes at most countable 2-multiple real roots.

Proof. Case 1.

Assume that $B_{2}=\varnothing$. Let $\xi_{0} \in(a, b)$ and $\left(\xi_{0}, x_{0}\right) \in A$. Since $f_{x}\left(\xi_{0}, x_{0}\right) \neq 0$, by Lemma 14 , the maximal path across $\left(\xi_{0}, x_{0}\right)$ must join with $P_{a}$ and $P_{b}$.

Case 2. Assume $B_{2} \neq \varnothing$. Let

$$
\bar{a}=\inf \left\{\xi \in B_{2}\right\}, \bar{b}=\sup \left\{\xi \in B_{2}\right\} .
$$

If $\bar{a}>a$ and $\bar{b}<b$, then $\bar{a}, \bar{b} \in B_{2}$. By Lemma 17 , there exists a path joining with $P_{\bar{a}}^{1}$ and $P_{\bar{b}}^{1}$. Moreover, the maximal path across $P_{\bar{a}}^{1}$ reaches to $\xi=a$ on the left and the maximal path across $P_{\bar{b}}^{1}$ reaches to $\xi=b$ on the right. It is easy to see that three paths on $[a, \bar{a}],[\bar{a}, \bar{b}]$, and $[\bar{b}, b]$ form a regular path between $\xi=a$ and $\xi=b$. Now we consider the case that $\bar{a}=a$ or $\bar{b}=b$.

Let $\bar{a}=a$. Take $\xi_{0} \in B_{2}$ and a sequence $\left\{a_{j}\right\} \subset B_{2}$ such that

$$
\xi_{0}=a_{0}>a_{1}>a_{2}>\cdots>a_{j}>a_{j+1} \longrightarrow a .
$$

Moreover, $P_{a_{j}}^{1} \longrightarrow P_{a}, P_{a_{j}}^{2} \longrightarrow P_{a}$. By Lemma 17, on $\left[a_{j-1}, a_{j}\right]$, we have a path $\ell_{j}$ joining with $P_{a_{j-1}}^{1}$ and $P_{a_{j}}^{1}$. Then, all the paths $\left\{\ell_{j}\right\}_{j \geq 0}$ can define a path joining with $P_{a_{0}}^{1}$ and $P_{a}$; for more details, we also refer to the proof of Lemma 26.

If $\bar{b}=b$, in the same way, we can define a path connected with $P_{a_{0}}^{1}$ and $P_{b}$. These two paths can be connected to form a regular path joining with $P_{a}$ and $P_{b}$.

Below, we consider the set of 3-multiple roots.

Lemma 19. For all $(\xi, x) \in A 3$, we have $x=x(\xi)=-\left(c_{1}(\xi)\right.$ / $3), \xi \in[a, b]$. That means, all 3-multiple real roots of $f$ are on the continuous curve

$$
l_{*}:\left\{(\xi, x) \mid x=-\frac{c_{1}(\xi)}{3}, a \leq \xi \leq b\right\} .
$$

Proof. Note that for $(\xi, x) \in A_{3}$, we have $f_{x x}(\xi, x)=6 x+2$ $c_{1}(\xi)=0$; the result holds obviously.

Now, we prove the following important lemma.

Lemma 20. There exists a regular path $\ell \subset A$ with two oddmultiple endpoints on $A(a)$ and $A(b)$, respectively.

Proof. We first claim that $B_{3}$ is a closed set. 
Let $\xi_{n} \longrightarrow \xi_{0}, \xi_{n} \in B_{3}$. Then, there exists a subsequence $x\left(\xi_{n_{k}}\right)$ of $x\left(\xi_{n}\right)$, such that $x\left(\xi_{n_{k}}\right) \longrightarrow x_{0}$. Since $f(\xi, x), f_{x}(\xi$, $x), f_{x x}(\xi, x)$ are continuous, by $\xi_{n_{k}} \longrightarrow \xi_{0}$ we have

$$
f\left(\xi_{0}, x_{0}\right)=f_{x}\left(\xi_{0}, x_{0}\right)=f_{x x}\left(\xi_{0}, x_{0}\right)=0 .
$$

Thus, $\xi_{0} \in B_{3}$ and so $B_{3}$ is closed.

Let $\bar{a}=\min \left\{\xi \mid \xi \in B_{3}\right\}, \bar{b}=\max \left\{\xi \mid \xi \in B_{3}\right\}$. Then, we have $[\bar{a}, \bar{b}] \backslash B_{3}=\cup_{j \geq 1}\left(a_{j}, b_{j}\right)$ where $\left\{\left(a_{j}, b_{j}\right) \mid j=1,2, \cdots\right\}$ are disjointed open intervals of each other.

By Lemma 18 , there exists a regular path $\ell_{j}$ between $\xi=a_{j}$ and $\xi=b_{j}$ such that $P_{a_{j}}, P_{b_{j}} \in \ell_{j}$.

Moreover, if $a<\bar{a}$, in the same way as the proof of Lemma 18 , where we take $\xi_{0}=a$ and $b=\bar{a}$, then there exists a regular path $\ell_{l}$, which joins with $P_{\bar{a}}$ and $P_{a}^{*}$, where $P_{a}^{*}$ is a simple root of $f(a, x)$. In the same way, if $\bar{b}<\mathrm{b}$, we have a regular path $\ell_{r}$, which joins with $P_{\bar{b}}$ and $P_{b}{ }^{*}$, where $P_{b}{ }^{*}$ is a simple root of $f(b, x)$. Now, we construct a path $\ell$ as follows:

$$
\begin{cases}l=l_{j}, & \xi \in\left(a_{j}, b_{j}\right), \quad j \geq 1, \\ l=l_{*}, & \xi \in B_{3}, \\ l=l_{l}, & \xi \in[a, \bar{a}], \\ l=l_{r}, & \xi \in[\bar{b}, b],\end{cases}
$$

where $l_{*}(\xi)=\left(\xi,-(1 / 3) c_{1}(\xi)\right)$. Thus, $\ell$ is a path joining with $P_{a}^{*}$ and $P_{b}^{*}$.

Note that if $a=\bar{a}$ (or $b=\bar{b}$ ), we have no path $\ell_{l}\left(\right.$ or $\ell_{r}$ ).

Below, we explain the continuity of $\ell$. Let $P_{0}=\left(\xi_{0}, x_{0}\right) \in$ $A_{3}$. By uniform continuity of continuous bifurcations of roots, it is easy to see that for any $\epsilon>0$, there exists $\delta>0$ such that if $\left|\xi-\xi_{0}\right| \leq \delta$ and $(\xi, x)$ is a real root of $f$, then $\left|x-x_{0}\right|$ $\leq \epsilon$. By this fact, the continuity of $\ell$ can be proven in the same way as in the extension of a continuous function on a closed set on $\mathbb{R}$ to $\mathbb{R}$; here, we omit the details.

Moreover, $\ell_{j}(j \geq 1), \ell_{l}, \ell_{r}$ include at most countable 2multiple real roots; thus, the path $\ell$ includes at most countable 2-multiple real roots.

Furthermore, by the above construction, it is easy to see that the path $\ell$ has no basis point. Then, $\ell$ is a regular path in $A$, which connects from $A(a)$ to $A(b)$.

3.5. $(2 d+1)$-Degree Polynomials. In this subsection, we will prove our main results in the general case. To make an induction, we first give some definitions.

Definition 21 (room of real roots and passage of odd-multiple real roots).

(1) Let $D=[a, b] \times(c, d)$ be a rectangle in the $(\xi, x)$-plane and $D^{\circ}=(a, b) \times(c, d)$ be the interior of $D$. Let $I_{0}=$ $\left[a_{0}, b_{0}\right] \subset \mathrm{I}=[a, b]$ and $D_{I_{0}}=I_{0} \times(c, d)$, where $D_{I}$ can be regarded as restriction of $D$ for $\xi$ to $I_{0}$. Denote the section of $D$ at $\xi$ by $D(\xi)=\{\xi\} \times(c, d) \subset \mathbb{R}^{2}$. In particular, $D(a)$ and $D(b)$ are called the left side and the right side of $D$, respectively
(2) Let $I=[a, b]$ and $D$ be a rectangle as given above. A group $(f, m, D, I)$ is called a room of real roots of $f$; it means that $f(\xi, x)$ is a real polynomial of degree $m$ with respect to $x$ and depends continuously in the parameter $\xi$ on $I$; moreover, all real roots of $f(\xi, x)$ fall into $D$ for all $\xi \in I$. If $f$ has an $m$ multiple real root on $D,(f, m, D, I)$ is called a minimal room of real roots of $f$

(3) If the left (right) side of $D$ contains an odd-multiple real root of $f$, the side is called a door of oddmultiple real roots. If a room of real roots of $f$ has a door of odd-multiple real roots, it is called a passage of odd-multiple real roots. Otherwise, if the left (right) side of $D$ contains no odd-multiple real root of $f$, the side is called a separating wall of odd-multiple real roots. If both the left side and the right side are separating walls of odd-multiple real roots, we call $(f, m, D, I)$ an insulator of odd-multiple real roots. Let $(f, 2 d+1, D, I)$ be a room of real roots. If $f(a, x)$ and $f(b, x)$ have $2 d+1$-multiple real roots, $(f, 2 d+$ $1, D, I)$ is called a one-way-passage of odd-multiple real roots

(4) If $D^{\circ}$ contains no any real root of $f,(f, m, D, I)$ is called a separating block of real roots

(5) If $(f, m, D, I)$ is a passage of odd-multiple real roots and $f$ has an $m$-multiple real root in $D$, we call $(f$, $m, D, I)$ a minimal passage of odd-multiple real roots. A minimal passage of odd-multiple real roots cannot be decomposed into smaller passages

A room of real roots is either a passage of odd-multiple real roots, or an insulator of odd-multiple real roots, or a separating block of real roots. Also, note that once a path enters into a one-way passage of odd-multiple real roots from one side, it can only go out of the one-way passage from the other side.

Note that an odd-multiple real root is never isolated and an even-multiple real root can be isolated. Thus, there are a lot of odd-multiple real roots near to a door of oddmultiple real roots, so a passage is always neighbouring with some rooms of real roots. A separating block of real roots has no use in our problem, so it is usually ignored.

Lemma 22 (decomposition of minimal rooms of real roots). Let $(f, m, D, I)$ be a room of real roots of $f$. Assume $f$ has no any $m$-multiple root for all $\xi \in I$. Then, there exists $\delta>0$, such that for any $\eta \in I$, if $f(\eta, x)$ has a real root, there exist real polynomials $f_{j}(\xi, x)(j=1,2)$, with their degrees $m_{j}<m$, which continuously depend on the parameter

$$
\xi \in I_{0}=[\eta-2 \delta, \eta+2 \delta] \cap I,
$$

and disjointed rectangles $D_{j} \subset D, j=1,2$, such that

$$
f(\xi, x)=f_{1}(\xi, x) f_{2}(\xi, x), \quad \xi \in I_{0},
$$


and all real roots of $f_{j}$ fall into $D_{j}, j=1,2$. Moreover, $f$ and $f_{j}$ have the same real roots on $D_{j}, j=1,2$.

Remark 23. In brief, the above statement can be written as

$$
\left(f, m, D_{I_{0}}, I_{0}\right)=\left(f_{1}, m_{1} D_{1}, I_{0}\right) \times\left(f_{2}, m_{2}, D_{2}, I_{0}\right) .
$$

Let $(f, m, D, I)$ be a room of real roots. If $\left(f_{j}, m_{j}, D_{j}, I_{0}\right)$ is a room of real roots, we call it a subroom of real roots of $(f, m, D, I)$. Similarly, we can define subpassage.

Also note that $\left(f_{j}, m_{j}, D_{j}, I_{0}\right)$ may not be a passage of odd-multiple real roots. In fact, $\left(f_{j}, m_{j}, D_{j}, I_{0}\right)$ may be a separating block of real roots, which is usually ignored in our problem.

Proof. We first consider the case that $m=2 d+1$. Let $\left\{x_{j}(\xi)\right.$ $, 1 \leq j \leq m\}$ be $m$ continuous bifurcations of roots of $f$ for $\xi$ on $I$. Since $f$ has no $m$-multiple real root, it follows that there exists $\alpha>0$ such that

$$
\max _{i \neq j}\left|x_{j}(\xi)-x_{i}(\xi)\right| \geq(2 d+1) \alpha, \quad \forall \xi \in I
$$

Let $x_{j_{1}}(\eta)$ be a real root of $f(\eta, x)$. Then, there exist $\delta>$ $0, c \geq 0,1 \leq r<m$ and two groups $\left\{x_{j_{1}}, \cdots, x_{j_{r}}\right\}$ and $\left\{x_{j_{r+1}}, \cdots\right.$, $\left.x_{j_{m}}\right\}$ such that

$$
\begin{aligned}
& \left|x_{j_{k}}(\xi)-x_{j_{1}}(\eta)\right|<c, \quad \forall \xi \in I_{0}, \forall 1 \leq k \leq r<m, \\
& \left|x_{j_{k}}(\xi)-x_{j_{1}}(\eta)\right|>c+\alpha, \quad \forall \xi \in I_{0}, \forall r+1 \leq k \leq m,
\end{aligned}
$$

where $I_{0}=[\eta-2 \delta, \eta+2 \delta] \cap I$. Since all $x_{j}(\xi)$ are uniformly continuous in $\xi$ on $I$, so $\delta$ can be independent of $\eta \in I$.

Note that for $r+1 \leq k \leq m$, if $\left|\operatorname{Re}\left(x_{j_{k}}(\xi)\right)-x_{j_{1}}(\eta)\right|<$ $\mathrm{c}+\alpha$, then $x_{j_{k}}(\xi)$ is a complex root.

Let $x_{0}=x_{j_{1}}(\eta)$ and $M$ be a constant such that $\left|x_{j}(\xi)\right| \leq$ $M, \forall \xi \in I, j=1,2, \cdots m$.

Now we divide $\left\{x_{j_{r+1}}, \cdots x_{j_{m}}\right\}$ into two groups:

$$
\begin{aligned}
& \left\{x_{j_{k}} \mid \operatorname{Re}\left(x_{j_{k}}\right) \leq x_{0}, r+1 \leq k \leq m\right\}=\left\{\tilde{x}_{j} \mid 1 \leq j \leq \tilde{r} \leq m-r\right\}, \\
& \left\{x_{j_{k}} \mid \operatorname{Re}\left(x_{j_{k}}\right)>x_{0}, r+1 \leq k \leq m\right\}=\left\{\tilde{x}_{j} \mid \tilde{r}+1 \leq j \leq m-r\right\} .
\end{aligned}
$$

It is easy to see that for $1 \leq j \leq \tilde{r}$, if $\tilde{x}_{j}$ is a real root, then $\tilde{x}_{j} \leq x_{0}-c-\alpha$, and for $\tilde{r}+1 \leq j \leq m-r$, if $\tilde{x}_{j}$ is a real root, then $\tilde{x}_{j}>\mathrm{x}_{0}+c+\alpha$.
Let

$$
\begin{aligned}
& g_{1}(\xi, x)=\left(x-x_{j_{1}}(\xi)\right) \cdots\left(x-x_{j_{r}}(\xi)\right), \\
& g_{2}(\xi, x)=\left(x-\tilde{x}_{1}(\xi)\right) \cdots\left(x-\tilde{x}_{\tilde{r}}(\xi)\right) \\
& g_{3}(\xi, x)=\left(x-\tilde{x}_{r+1}(\xi)\right) \cdots\left(x-\tilde{x}_{m-r}(\xi)\right) .
\end{aligned}
$$

Since $x_{j_{1}}(\eta)$ is a real number, it follows that $x_{j_{k}}(\xi), \tilde{x}_{j}(\xi)$, and their conjugate complex roots belong to the same group; thus, all $g_{j}(j=1,2,3)$ are real polynomials.

If $\tilde{r} \geq 1$, let

$f_{1}=g_{1} g_{3}, f_{2}=g_{2}, D_{1}=I_{0} \times\left(-M, x_{0}+c\right), D_{2}=I_{0} \times\left[x_{0}+c+\alpha, M\right]$.

If $\tilde{r}=0$, let

$f_{1}=g_{1}, f_{2}=g_{3}, D_{1}=I_{0} \times\left(x_{0}-c, x_{0}+c\right), D_{2}=I_{0} \times\left(-M, x_{0}-c-\alpha\right)$.

The case that $m=2 d$ can be considered in the same way by taking $x_{0}=x_{j_{1}}(\eta)$ to be a real root of $f(\eta, x)$, which is available by assumption.

If $f$ has an $m$-multiple real root, the room of real roots $(f, m, D, I)$ cannot be decomposed into smaller ones.

The fact that $\delta$ is independent of $\eta$ guarantees a finite partition of $[a, b]$ such that, on each small interval, we have the above decomposition. If some smaller room is not minimal, we can use the above lemma again until all small rooms are minimal. This result concerns with the following lemma.

Lemma 24. Consider $\left(f_{0}, 2 d+1, D_{0}, I_{0}\right)$ and let $(f, m, D, I)$ with $I=[a, b]$ be a subroom of $\left(f_{0}, 2 d+1, D_{0}, I_{0}\right)$ with $m \leq$ $2 d+1, D \subset D_{0}, I \subset I_{0}$. Assume that $f$ and $f_{0}$ have same real roots on $D$; moreover, $f$ has no any m-multiple root for all $\xi \in[a, b]$. Then, there exists finitely many minimal rooms of real roots of $f,\left\{\left(f_{j}, m_{j}, D_{j}, I_{j}\right)\right\}_{j=1}^{N}$ with $I_{j}=\left[a_{j}, b_{j}\right]$, such that the following hold true:

(1) $f$ and $f_{j}$ have same real roots on $D_{j}$

(2) $m_{j}<m$ and $f_{j}$ has at least one $m_{j}$-multiple real root on $D_{j}$

(3) If $a_{j} \neq a$ and the left side $D_{j}\left(a_{j}\right)$ contains a real root $P$ of $f_{j}$, then there is a room of real roots $\left(f_{i}, m_{i}, D_{i}, I_{i}\right)$, which is neighboured with $\left(f_{j}, m_{j}, D_{j}, I_{j}\right)$ such that $P$ $\in \stackrel{\circ}{D}_{i}$ and $a_{i}<a_{j}<b_{i}$. For the right side, if $b_{j} \neq b$ and $D_{j}\left(b_{j}\right)$ contains a real root of $f_{j}$, we have a similar result. Let $\delta_{i j}=\min \left\{\left|a_{i}-b_{j}\right|,\left|b_{i}-b_{j}\right|\right\}$, then we have $\delta_{i j}>0$. Since $i, j \leq N$, the smallest distance of such $\delta_{i j}$ is also positive 
(4) Every $\left(f_{j}, m_{j}, D_{j}, I_{j}\right)$ is a minimal passage of oddmultiple real roots or an insulator of odd-multiple real roots; moreover, any real root of $f$ on $D$ is a real root of some $f_{j}$ on $D_{j}$

The conclusion (3) is very important in extending of paths. By induction, a path can be always extended from an initial point in a passage of real roots $D_{j}$ to arrive at an odd-multiple real root on a door of $D_{j}$. The new oddmultiple endpoint on the door of $D_{j}$ is already in the interior of some $D_{i}$. Then, we can do extension again in $D_{i}$ to arrive at another odd-multiple endpoint on a door of $D_{i}$; in this process, the path must cross at least $\delta_{i j}$ length of $\xi$. Since a path can only cross at most $(2 d+1)(b-a)$ length about the parameter, the extension can repeat at most finitely many times before it cannot be extended again, which can only happen when the path has become a closed regular path or it arrives the boundary of the domain.

Among the sequence of minimal rooms $\left\{\left(f_{j}, m_{j} D_{j}, I_{j}\right)\right\}_{j=1}^{N}$ in this lemma, there may be some minimal room which is completely included in a bigger one. In this case, we can drop away some smaller minimal rooms and the lemma still holds for the rest. So, without loss of generality, we can suppose $D_{i} \backslash D_{j} \neq \varnothing$ for all $i \neq j$.

Proof of Lemma 24. By assumption, $f$ has no any $m$-multiple real root. By Lemma 22, there exists $\delta>0$ such that for $\eta \in[a, b]$, if $f(\eta, x)$ has a real root, then there exist two real polynomials $f_{1}(\xi, x)$ and $f_{2}(\xi, x)$ with their degrees $m_{1}$, $m_{2}<\mathrm{m}$, which continuously depend on the parameter $\xi \in[\eta-2 \delta, \eta+2 \delta] \cap[a, b]$, and two disjointing rectangles $D_{1}$ and $D_{2}$ for $\xi$ on $[\eta-2 \delta, \eta+2 \delta] \cap[a, b]$, such that $f(\xi, x)=f_{1}(\xi, x) f_{2}(\xi, x)$ and all real roots of $f_{i}$ fall into $D_{i}, \quad i=1,2$.

Now, we choose a partition of $[a, b]$ :

$$
a=\xi_{0}<\xi_{1}<\xi_{2}<\cdots<\xi_{n}=b
$$

such that $\xi_{j+1}-\xi_{j}<\delta$. Then, let

$$
\left\{I_{j}^{1}=\left[\xi_{j}, \xi_{j+2}\right] \mid j=0,1, \cdots n-2\right\} .
$$

If $f(\eta, x)$ has a real root for some $\eta \in\left(\xi_{j}, \xi_{j+2}\right)$, Lemma 22 implies that on the small interval $I_{j}^{1}$, we have the decomposition $\left(f_{j 1}^{(1)}, m_{j 1}^{(1)}, D_{j 1}^{(1)}, I_{j}^{(1)}\right)$ and $\left(f_{j 2}^{(1)}, m_{j 2}^{(1)}, D_{j 2}^{(1)}, I_{j}^{(1)}\right)$.

If $f(\eta, x)$ has no any real root for all $\eta \in\left(\xi_{j}, \xi_{j+2}\right)$, we drop away the room of real roots $\left(f, m, D_{I_{j}^{1}}, I_{j}^{(1)}\right)$. In this case, if there exists a real root $P$ on $D\left(\xi_{j}\right)$ or $D\left(\xi_{j+2}\right), P$ is an evenmultiple real root and belongs to $\left(f, m, D_{I_{j-1}^{1}}, I_{j-1}^{1}\right)$ or $(f, m$, $\left.D_{I_{j+1}^{1}}, I_{j+1}^{1}\right)$, so we do not lose any real root after dropping away $\left(f, m D_{I_{j}^{1}}, I_{j}^{(1)}\right)$.
Denote all these smaller rooms of real roots by $\left\{\left(f_{j}^{(1)}\right.\right.$, $\left.\left.m_{j}^{(1)}, D_{j}^{(1)}, I_{j}^{(1)}\right)\right\}$ for simplicity of notations. Note that some two intervals in $\left\{I_{j}^{(1)}\right\}$ may be identical, but $\left\{D_{j}^{(1)}\right\}$ are all different from each other. Let $I_{j}^{(1)}=\left[a_{j}^{(1)}, b_{j}^{(1)}\right]$.

If some $f_{j}^{(1)}$ has no real root in the interior of $D_{j}^{(1)}$, that is, $\left(f_{j}^{(1)}, m_{j}^{(1)}, D_{j}^{(1)}, I_{j}^{(1)}\right)$ is a separating block of real roots, we drop it away. So without loss of generality, assume $f_{j}^{(1)}$ has at least one real root in the interior of $D_{j}$. Thus, $\left(f_{j}, m_{j}, D_{j}\right.$, $\left.I_{j}\right)$ is a passage of odd-multiple real roots or an insulator of odd-multiple real roots. After dropping some separating blocks, for simplicity, we denote the subsequence by itself

$$
\left\{\left(f_{j}^{(1)}, m_{j}^{(1)}, D_{j}^{(1)}, I_{j}^{(1)}\right)\right\}_{j=1}^{N^{1}} .
$$

It is very important that for any side of $D_{j}^{(1)}$, say $D_{j}^{(1)}\left(a_{j}^{(1)}\right)$, if $a_{j}^{(1)} \neq a$ and $P \in D_{j}^{(1)}\left(a_{j}^{(1)}\right)$ is a real root, then $P$ must belong to the interior of some $D_{i}^{(1)}(i \neq j)$. If $b_{j}^{(1)} \neq b$ and $P \in D_{j}^{(1)}\left(b_{j}^{(1)}\right)$ is a real root, we have a similar result.

Now, if $\left(f_{1}^{(1)}, m_{1}^{(1)}, D_{1}^{(1)}, I_{1}^{(1)}\right)$ is not minimal, in the same way as above, we decompose it into a sequence of smaller rooms of real roots

$$
\left\{\left(f_{1 k}^{(2)}, m_{1 k}^{2}, D_{1 k}^{(2)}, I_{1 k}^{(2)}\right)\right\}_{k \geq 1}
$$

But we should note that when we make a partition of $I_{1}^{(1)}=\left[a_{1}^{(1)}, b_{1}^{(1)}\right]$, we require the points of the partition not to fall on $M_{1}$, where

$$
M_{1}=\left\{\xi \mid D_{1}^{(1)} \cap D_{j}^{(1)}(\xi) \neq \phi, \xi \in\left\{a_{j}^{(1)}, b_{j}^{(1)}\right\}, 1 \leq j \leq N_{1}\right\} .
$$

Since $M_{1}$ is finite set, it is easy to have such partition of $\left[a_{1}^{(1)}, b_{1}^{(1)}\right]$. Such partition is very important, and it makes the sides of a neighbouring room of real roots to disjoint each other. Thus, any real root on a side must fall into the interior of a neighbouring room unless it falls into $D(a) \cup D(b)$.

If $\left(f_{1}^{(1)}, m_{1}^{(1)}, D_{1}^{(1)}, I_{1}^{(1)}\right)$ is minimal, we cannot decompose it and denote it by

$$
\left\{\left(f_{11}^{(2)}, m_{11}^{(2)}, D_{11}^{(2)}, I_{11}^{(2)}\right)=\left(f_{1}^{(1)}, m_{1}^{(1)}, D_{1}^{(1)}, I_{1}^{(1)}\right)\right\}
$$

Suppose we have decomposed

$$
\left\{\left(f_{j k}^{(1)}, m_{j k}^{(1)}, D_{j k}^{(1)}, I_{j k}^{(1)}\right)\right\}, \quad j=1,2, \cdots i-1,
$$

and obtained

$$
\left\{\left(f_{j k}^{(2)}, m_{j k}^{(2)}, D_{j k}^{(2)}, I_{j k}^{(2)}\right)\right\}, \quad j=1,2, \cdots i-1 .
$$


Now, we consider $\left(f_{i}^{(1)}, m_{i}^{(1)}, D_{i}^{(1)}, I_{i}^{(1)}\right)$. In the same way, we make a partition of $\left[a_{i}, b_{i}\right]$ as above; moreover, we require the points of the partition not to fall on $M_{i}$, where $M_{i}=$ $M_{i}^{1} \cup M_{i}^{2}$, where

$$
\begin{aligned}
& M_{i}^{1}=\left\{\xi \mid D_{i}^{(1)} \cap D_{j}^{(1)}(\xi) \neq \phi, \xi \in\left\{a_{j}^{(1)}, b_{j}^{(1)}\right\}, j>i\right\} \\
& M_{i}^{2}=\left\{\xi \mid D_{i}^{(1)} \cap D_{j}^{(2)}(\xi) \neq \phi, \xi \in\left\{a_{j}^{(2)}, b_{j}^{(2)}\right\}, j=i, \cdots i-1, k \geq 1\right\} .
\end{aligned}
$$

If $\left(f_{i}^{(1)}, m_{i}^{(1)}, D_{i}^{(1)}, I_{i}^{(1)}\right)$ is minimal, we cannot decompose it and denote it by

$$
\left\{\left(f_{i 1}^{(2)}, m_{i 1}^{(2)}, D_{i 1}^{(2)}, I_{i 1}^{(2)}\right)=\left(f_{i}^{(1)}, m_{i}^{(1)}, D_{i}^{(1)}, I_{i}^{(1)}\right)\right\}
$$

In this way, we decompose all rooms $\left(f_{j}^{(1)}, m_{j}^{(1)}, D_{j}^{(1)}\right.$, $\left.I_{j}^{(1)}\right)$ which are not minimal.

Then, we move away all separating blocks from $\left\{\left(f_{j k}^{(2)}\right.\right.$, $\left.\left.m_{j k}^{(2)}, D_{j k}^{(2)}, I_{j k}^{(2)}\right)\right\}$, and the rest is denoted by

$$
\left\{\left(f_{j}^{(2)}, m_{j}^{(2)}, D_{j}^{(2)}, I_{j}^{(2)}\right)\right\}_{j=1}^{N^{2}} .
$$

This step can iterate at most $m$ steps, and finally, we have a sequence of minimal rooms of real roots

$$
\left\{\left(f_{j}^{(m)}, m_{j}^{(m)}, D_{j}^{(m)}, I_{j}^{(m)}\right)\right\}_{j=1}^{N^{m}} .
$$

For simplicity, denote it by

$$
\left\{\left(f_{j}, m_{j}, D_{j}, I_{j}\right)\right\}_{j=1}^{N} \text {. }
$$

According to the above decomposition, it is easy to see that if one side of $\left(f_{j}, m_{j}, D_{j}, I_{j}\right)$ does not fall on $\xi=a$ or $\xi=b$, it may be divided into some parts such that each part is between the two sides of a neighbouring room $\left(f_{i}, m_{i}\right.$, $\left.D_{i}, I_{i}\right)$, the overlapping region $D_{j} \cap D_{i}$ can be regarded as a door way joining with $\left(f_{j}, m_{j}, D_{j}, I_{j}\right)$ and $\left(f_{i}, m_{i}, D_{i}, I_{i}\right)$. Any real root on the side of $D_{j}$ is in the interior of some $D_{i}$ with $i \neq j$.

This is very important for our extension of paths. By induction assumption, a path of odd-multiple real roots can be extended in $D_{j}$ to a side of $D_{j}$. Since the new oddmultiple endpoint on the side of $D_{j}$ falls into the interior of some $D_{i}$, we can extend the path in $D_{i}$ again from the new endpoint to a side of $D_{i}$. In this way, we can extend a path from the initial endpoint in some room to a neighbouring room and then go to the next neighbouring room. This process can go on unless it has become a closed regular path or arrived on $\xi=a$ or $\xi=b$.
We should note that once a path enters into an insulator of odd-multiple real roots, the whole path belongs to the insulator. Moreover, any extension of the path can only stay in the insulator forever and it can only be extended to be a closed path finally. So, only in some passages of oddmultiple real roots a path can be extended to be a desired path.

A one-way passage of odd-multiple real roots can only allow one regular path to go through it. Once a segment of a path goes from $D(a)$ to $D(b)$, since there is a unique real root on $D(a)$ and $D(b)$, respectively, according to the compatibility of paths, any segment of the path cannot enter into it again.

We also note that a bigger room may be neighbouring with several smaller rooms. This case happens near to some multiple real roots, where some paths of lowerodd-multiple real roots bifurcate.

The proof of Theorem 2 is based on a special case that $f$ has at least one $(2 d+1)$-multiple real root, which is considered in the following theorem:

Theorem 25. Consider a room of real roots $(f, 2 d+1, D, I)$ with $I=[a, b]$. If $f$ has $a(2 d+1)$-multiple real root, then there exists a regular path of odd-multiple real roots in $D$, which joins with two odd-multiple endpoints on $D(a)$ and $D(b)$, respectively.

The proof of Theorem 25 is based on induction for degree of polynomials. So we first assume Theorem 25 already holds for polynomials of degree $\leq 2 d-1$. Below, we prove that Theorem 25 also holds for polynomials of degree $=2 d+1$. The proof is based on the following lemma.

Before giving the lemma, we first show some notations. Let $\tilde{m} \leq 2 d+1$ and $(f, \tilde{m}, D, I)$ be a room of real roots of $f$ with $I=[a, b]$. Let $A_{\tilde{m}}$ be the set which consists of all $\tilde{m}$-multiple real roots of $f$ for all $\xi \in I$ and $B_{\tilde{m}}=\left\{\xi \in I \mid(\xi, x) \in A_{\tilde{m}}\right\}$. For $\xi \in$ $B_{\tilde{m}}$, denote by $P_{\xi}$ the $\tilde{m}$-multiple real root of $f(\xi, x)$.

Lemma 26. Let $(f, \tilde{m}, D, I)$ be a subroom of real roots of $\left(f_{0}, 2 d+1, D_{0}, I_{0}\right)$. Let $\gamma_{0}$ be a regular path in $\left(f_{0}, 2 d+1\right.$, $\left.D_{0}, I_{0}\right)$ with an odd-multiple endpoint $P_{0} \in D_{0} \backslash D$ and $a$ simple odd-multiple endpoint $P_{1} \in D_{0}$. Let $\tilde{\gamma}_{j}, \cdots \tilde{\gamma}_{k}$ be paths in $\left(f_{0}, 2 d+1, D_{0}, I_{0}\right)$, where $k \leq d$.Let $E_{j}=\left\{\tilde{\gamma}_{j}(0), \tilde{\gamma}_{j}(1)\right\}$ be the endpoints of $\tilde{\gamma}_{j}$. Suppose that $\tilde{\gamma}_{j}$ is a closed regular path or $E_{j} \subset D_{0} \backslash D$. Let $\tilde{\gamma}=\tilde{\gamma}_{1} \cup \cdots \cup \tilde{\gamma}$ and $\Gamma=\gamma_{0} \cup \tilde{\gamma}$. Suppose that $\left\{\gamma_{0}, \tilde{\gamma}_{1}, \cdots \tilde{\gamma}_{k}\right\}$ are compatible on $D$.

(1) Let $\tilde{m}=2 \bar{d}$ and $B_{2 \bar{d}}=\varnothing$. Suppose that $\gamma_{0}$ enters into $D$ with $P_{1} \in D^{\circ}$. Then $\gamma_{0}$ can be extended in $D$ to be a compatible path such that it has a simple oddmultiple endpoint $P_{1+}$ on $D(a) \cup D(b)$ with outward direction. Here "outward direction" means, if $P_{1+} \in$ $D(a)($ or $D(b))$, it is a left (or right) endpoint

Note that below the new endpoint in extension of paths always has outward direction in the above sense if without explanation.

(2) Let $\tilde{m}=2 \bar{d}$ and $P_{1} \in D^{\circ}$. Then, the following hold true: 
(i) Let $B_{2 \bar{d}}=\{b\}$. Suppose that $\gamma_{0}$ enters into $D$ and arrives at $P_{1}=\left(c, x_{c}\right)$ with $a<c<b$. Then, $\gamma_{0}$ can be extended in $D$ to be a compatible path such that it has a simple odd-multiple left endpoint on $\mathrm{D}(a)$, or it reaches to $P_{b}$. Moreover, in the last situation, we can continue to extend $\gamma_{0}$ with $P_{b}$ as a reflecting point such that it has an odd-multiple left endpoint on $D(a)$; moreover, it is compatible with $\tilde{\gamma}$

(ii) Let $B_{2 \bar{d}} \neq \varnothing$. Then, $\gamma_{0}$ can be extended to be a compatible path such that it has a simple oddmultiple endpoint on $D(a)$ or $D(b)$ with outward direction

(3) Let $\tilde{m}=2 \bar{d}+1$ and $B_{2 \bar{d}+1}=\varnothing$. Suppose that $\gamma_{0}$ enters into $D$ with $P_{1} \in D^{\circ}$. Then, $\gamma_{0}$ can be extended to be a compatible path such that it has a simple odd-multiple endpoint on $D(a)$ or $D(b)$ with outward direction

(4) Let $\tilde{m}=2 \bar{d}+1$ and $B_{2 \bar{d}+1}=\{b\}$. Then, the following hold true:

(i) Suppose that $\gamma_{0}$ enters into $D$ with $P_{1}=P_{c}=$ $\left(c, x_{c}\right) \in D$ with $a<c<b$. Then, it can be extended to be a compatible path such that it has an oddmultiple left endpoint on $D(a)$, or it arrives at $P_{b}$. Moreover, in the last situation, if $P_{b}$ is already $a$ crossing point, $\gamma_{0}$ can be extended to be a compatible path such that it has an odd-multiple left endpoint $P_{1+}$ on $D(a)$

(ii) Cancel the existing paths $\gamma_{0}$ and $\tilde{\gamma}_{j}$ in the assumption and consider $(f, \tilde{m}, D, I)$ to be an empty room of real roots. Then, there exists a regular path $\gamma \subset D$ which joins with oddmultiple endpoints $P_{0} \in D(a)$ and $P_{1}=P_{b}$ with outward direction

(5) Let $\tilde{m}=2 \bar{d}+1$ and $B_{2 \bar{d}+1}=\{a, b\}$. Then, the following hold true:

(i) Suppose that the path $\gamma_{0}$ reaches to $D$ with $P_{1} \in D$. Then, the path $\gamma_{0}$ can be extended to be a compatible path such that it has a simple endpoint belonging to $\left\{P_{a}, P_{b}\right\}$ with outward direction

(ii) In the same way as (4(ii)), there exists a regular path $\gamma \subset D$, which joins with endpoints $P_{a}$ and $P_{b}$ with outward direction.

(6) Let $\tilde{m}=2 \bar{d}+1$ and $B_{2 \bar{d}+1} \neq \varnothing$. Then, the following hold true:

(i) Let $\bar{d} \leq d-1$. Suppose that $\gamma_{0}$ enters into $D$ with $P_{1} \in D^{\circ}$. Then, $\gamma_{0}$ can be extended to be a compatible path such that it has a simple odd-multiple endpoint on $D(a) \cup D(b)$ with outward direction

(ii) Let $\bar{d}=d$. Then, Theorem 25 holds true
Remark 27.

(i) If $B_{2 \bar{d}}=\{a\}$, we have similar results to (2(i)) and (2(ii)). Moreover, if $B_{2 \bar{d}+1}=\{a\}$, we have similar results to (4(i)) and (4(ii)). Here, we omit the details

(ii) We require endpoints of $\tilde{\gamma}_{j}$ to be outside of $D$ to avoid $\gamma_{0}$ being in a dilemma after it enters into $D$. Thus, when $\gamma_{0}$ goes into $D$, it can always have some way to arrive at $D(a)$ or $D(b)$

Proof. We prove Lemma 26 by induction. By the proof of Lemma 20, it is easy to see that Lemma 26 holds for $\bar{d}=1$. Inductively suppose that Lemma 26 holds for all $\bar{d} \leq m-1$. Below, we prove it for $\bar{d}=m$. Recall that we already make an induction assumption about $d$ for Theorem 25. Here, we actually make an induction about both $d$ and $\bar{d}$ in Theorem 25 and Lemma 26.

Proof of (1). By assumption, we have $B_{2 \bar{d}}=\varnothing$. Then, by Lemma 24 , it follows that the room $(f, 2 \bar{d}, D,[a, b])(\bar{d}=m)$ can decompose into smaller minimal ones $\left\{\left(f_{j}, m_{j}, D_{j}, I_{j}\right)\right\}$ with $m_{j}<2 m$.

Suppose that the path $\gamma_{0}$ has been extended to some room $\left(f_{j}, m_{j}, D_{j}, I_{j}\right)$ such that $P_{1} \in \stackrel{\circ}{D}_{j}$. By assumption it is easy to see that $E=\cup_{j=1}^{k} E_{j}$, the set of all the endpoints of $\left\{\tilde{\gamma}_{j}\right\}$, is outside of $D_{j}$. In addition, whatever $m_{j}=2 \bar{m}$ or $m_{j}=2 \bar{m}+1$, we have $\bar{m} \leq m-1$. By induction assumption of (2(ii)) and (6), we can extend $\gamma_{0}$ in $D_{j}$ to a side of $D_{j}$ with a new odd-multiple left (or right) endpoint on $D_{j}\left(a_{j}\right)$ (or $\left.D_{j}\left(b_{j}\right)\right)$.

Moreover, we can require the new odd-multiple endpoint to be simple and have outward direction about domain $D_{j}$. In fact, we can always extend the path to reach to $D_{j}\left(a_{j}\right)$ (or $D_{j}\left(b_{j}\right)$ ) such that the endpoint is neither a crossing point nor a reflecting point. If not so, the endpoint meets a crossing point or a reflecting point on $D_{j}\left(a_{j}\right)$ (or $\left.D_{j}\left(b_{j}\right)\right)$, we can always continue the extension by reflecting at the crossing point or reflecting point such that the path enters into $D_{j}$ again and then repeat the above process.

If the endpoint is already on $D(a) \cup D(b)$, the result is proved. Otherwise, the odd-multiple endpoint is on a door of the passage $\left(f_{j}, m_{j}, D_{j}, I_{j}\right)$, which must be neighboured with another room of real roots, say, $\left(f_{i}, m_{i}, D_{i}, I_{i}\right)$. Then, the path enters into $D_{i}$ and the odd-multiple endpoint on the door of $\left(f_{j}, m_{j}, D_{j}, I_{j}\right)$ belongs to the interior of $D_{i}$; thus, we can do extension in $\left(f_{i}, m_{i}, D_{i}, I_{i}\right)$ in the same way as in $\left(f_{j}, m_{j}, D_{j}, I_{j}\right)$.

If the extension does not go to $D(a) \cup D(b)$, the process can repeat in the next neighbouring room. Each extension must go across the parameter of a positive length $\delta>0$. It is easy to see that the total length of parameter of $\gamma$ is no more 
than $2 \bar{d}(b-a)$; thus, after at most finitely many extensions, the path must reach to $D(a) \cup D(b)$ with an odd-multiple endpoint with outward direction.

Thus, we have $n$ segments of paths $\gamma_{1}, \cdots, \gamma_{n}$ such that

$P_{0}=\gamma_{1}(0), P_{1}=\gamma_{1}(1), \cdots, \gamma_{n-1}(1)=P_{n-1}=\gamma_{n}(0), P_{n}=\gamma_{n}(1)$,

where $P_{0}$ is the initial endpoint of the path.

Note that each $\gamma_{j}$ is obtained when we do extension in some passage. Moreover, each $\gamma_{j}$ is compatible with the existing paths since the extension is done according to the compatibility of paths by induction assumption. We should also note that once a path enters into an insulator, the path must totally fall into this insulator. However, since $P_{0} \notin D$, this situation cannot happen in our extension. Anyway, by induction assumption, the extension is well defined.

Let $I_{j}=[i /(n+1),(j+1) /(n+1)], j=0,1, \cdots n$ and $[0,1]$ $=\cup_{j=0}^{n} I_{j}$. By rescaling the parameter $t$ of paths, we suppose that the original path $\gamma_{0}(t)$ is defined for $t$ on $I_{0}$, and $\gamma_{j}(t)$ is defined for $t$ on $I_{j}, j \geq 1$. Then, we obtain an extension $\gamma=\cup_{j=0}^{n} \gamma_{j}$ with

$$
\gamma(t)=\gamma_{j}(t), \quad t \in I_{j}, j=0,1 \cdots n .
$$

By the above discussion, every extension follows the compatibility of paths, so all the paths are compatible. Thus, conclusion (1) is proven.

Proof of (2). Now, we turn to proving conclusion (2).

Case of (2(i)). (A) We first prove that once $\gamma_{0}$ enters into $D$, it can always be extended to arrive at $D(a)$ or $P_{b}$; moreover, it is compatible with $\tilde{\gamma}=\{\tilde{\gamma} \mid j=1,2 \cdots k\}$.

Take a partition

$$
a=a_{0}<c<a_{1}<\cdots<a_{n}<\cdots b \text { such that } a_{n} \longrightarrow b .
$$

Since $f$ has no $2 \bar{d}$-multiple real root for $\xi \in\left[a_{0}, a_{1}\right]$, by induction assumption and using the result (1) to the room of real roots $\left(f, 2 \bar{d}, D_{\left[a_{0}, a_{1}\right]}\left[a_{0}, a_{1}\right]\right)$, we can extend $\gamma_{0}$ such that it goes to $D(a) \cup D\left(a_{1}\right)$. If it arrives at $D(a)$, the result is proved; otherwise, it goes to $D\left(a_{1}\right)$. Denote the segment of extension by $\ell_{1}$. Note that here we can require the endpoint to be a simple one as discussed above; moreover, $\ell_{1}$ is compatible with $\Gamma=\tilde{\gamma} \cup \gamma_{0}$.

Let $\gamma_{1}=\gamma_{0} \cup \ell_{1}$, then $\gamma_{1}$ is compatible with $\tilde{\gamma}$. Now, we extend the path $\gamma_{1}$ in $D_{\left[a_{0}, a_{2}\right]}$ with a simple odd-multiple endpoint on $D\left(a_{1}\right)$ as a new initial endpoint. In the same way as above, if it does not arrive at $D(a)$, it goes to $D\left(a_{2}\right)$. Denote the segment of the extension by $\ell_{2}$. Moreover, $\ell_{2}$ is compatible with $\Gamma_{1}=\tilde{\gamma} \cup \gamma_{1}$. Let $\gamma_{2}=\gamma_{1} \cup \ell_{2}$, then $\gamma_{2}$ is compatible with $\tilde{\gamma}$.

Then, we repeat the above process of extension. If the path is already extended to $\xi=a_{n}$, we consider $\left[a_{0}, a_{n+1}\right]$. In the same way, if it does not arrive at $D(a)$, it must arrive at $\xi=a_{n+1}$.

In this process, if the path does not arrive at $D(a)$, it must arrive at all $\xi=a_{n}$ and have a sequence of segments of paths $\left\{l_{n}\right\}$, which is obtained when extending on $\left[a_{0}, a_{n}\right]$ and given by a continuous mapping $\ell_{n}: t \in[0,1] \longrightarrow \ell_{n}(t)$ with $P_{n}=$ $\ell_{n}(0) \in D\left(a_{n}\right)$ and $P_{n+1}=\ell_{n}(1) \in D\left(a_{n+1}\right)$, where $P_{n}$ are all simple odd-multiple right endpoints of $\ell_{n-1}$. Let

$$
[0,1]=\cup_{n=1}^{\infty}\left[1-\frac{1}{n}, 1-\frac{1}{n+1}\right) \cup\{1\}
$$

By rescaling the curve parameter $t$, we can let $\gamma_{0}(t)$, $t \in[0,1 / 2]$ and $\ell_{n}: t \in[1-1 /(n+1), 1-1 /(n+2)] \longrightarrow \ell_{n}(t)$ with $P_{n}=\ell_{n}(1-1 /(n+1))$ and $P_{n+1}=\ell_{n}(1-1 /(n+2))$.

Define

$\widehat{\gamma}: \widehat{\gamma}(t)=\ell_{n}(t), t \in\left[1-\frac{1}{n+1}, 1-\frac{1}{n+2}\right), \quad \forall n \geq 1, \widehat{\gamma}(1)=P_{b}$.

Then, $\hat{\gamma}(t), t \in[1 / 2,1]$ is a continuous mapping with $\widehat{\gamma}(0)=P_{1}$ and $\hat{\gamma}(1)=P_{b}$. So $\gamma=\gamma_{0} \cup \widehat{\gamma}$ is a continuous curve for $t \in[0,1]$ joining with $P_{0}$ and $P_{b}$.

We should note that in the above extension, $P_{b}$ may be already a reflecting point of $\tilde{\gamma}_{j}$ or $\gamma_{0}$. In this case, we can regard $\tilde{\gamma}_{j}$ as two segments of paths with $P_{b}$ as their endpoints. Obviously, all their endpoints are outside of $D_{\left[a_{0}, a_{n}\right]}$ for all $n \geq 1$. Moreover, $\Gamma$ is still compatible when $\tilde{\gamma}_{j}$ is divided into two pieces of paths. Then, the assumption of (1) holds, so the above extension is reasonable.

Below, we show that the extension $\gamma$ is well defined and satisfies the definition of paths; thus, $\gamma$ is a desired path.

By Lemma 11, since the points on a path can recur to some parameter at most $2 \vec{d}$ times, so $\ell_{n}$ can intersect with $\left\{\ell_{1}, \cdots, \ell_{n-1}\right\}$ at most $2 \bar{d}$ times. So for any $\ell_{m}$, there exists $N>m$ such that for all $n>\mathrm{N}, \ell_{n} \cap \ell_{m}=\varnothing$.

Let $\gamma_{n=} \gamma_{0} \cup\left(\cup_{k=1}^{n-1} \ell_{k}\right)$. By induction, all $\ell_{n}$ and $\gamma_{n}$ follow the compatibility of paths.

It follows that all $\gamma_{n}$ are compatible with $\tilde{\gamma}$.

Let $\gamma=\gamma_{0} \cup\left(\cup_{n=1}^{\infty} \ell_{n}\right) \cup\left\{P_{b}\right\}$. Thus, for any $P \in \gamma$, if $P \neq$ $P_{b}$, there exists sufficiently large $N>0$ such that $P \notin \cup_{n \geq N} \ell_{n}$ and $P \in \gamma_{N}=\gamma_{0} \cup\left(\cup_{n \leq N-1} \ell_{n}\right)$. By induction $\gamma_{N}$ is a welldefined compatible path, so $P$ satisfies the definition of path as a point on $\gamma_{N}$. Since $P \notin \cup_{n>N} \ell_{n}$, then $P$ also satisfies the definition of paths as a point on $\gamma$.

Below, we prove that $\gamma(t)$ is continuous at $t=1$. Note that $\gamma(1)=P_{b}$. Let $\gamma(t)=(\xi(t), x(t))$. For any $\epsilon>0$, there exists $N>0$ such that for all $n>N, \ell_{n}$ do not go across $\xi=b-\epsilon$. Note that $\ell_{n}=\left(\xi_{n}(t), x_{n}(t)\right), t \in[1-1 /(n+1), 1-1 /(n+2)]$. Then, for all $n>N, \xi_{n}(t) \in[b-\epsilon, b]$. Let $\delta=1 /(N+1)$. Then, if $0<1-t \leq \delta$, there exists $n>N$ such that $t \in[1-1 /(n+1)$, $1-1 /(n+2)]$, so $\xi(t)=\xi_{n}(t)$ and $|b-\xi(t)|=\left|b-\xi_{n}(t)\right| \leq \epsilon$. Thus, $\xi(t)$ is continuous at $t=1$. Moreover, since $P_{b}$ is a $2 \bar{d}$ 
-multiple real root, by the $2 \bar{d}$ continuous bifurcations of roots of the polynomial $f$, one of which the real $\operatorname{root}(\xi(t), x(t))$ must belong to; it follows easily that if $t<1$ and $t \longrightarrow 1$, we have $\xi(t) \longrightarrow b$ and so $x(t) \longrightarrow x_{b}$. Thus, $\gamma(t) \longrightarrow P_{b}, t \longrightarrow 1$, which implies that $\gamma(t)$ is continuous at $t=1$. Thus, $\gamma$ is a compatible path with $\tilde{\gamma}$, and $P_{b}$ is a left even-multiple endpoint.

(B) If $\gamma_{0}$ is already extended to arrive at $P_{b}$, we consider to extend it back from $P_{b}$ to $D(a)$. We prove that there exists a path $\hat{\gamma}$, which joins also from an odd-multiple real root $P_{1+} \in D(a)$ to $P_{b}$, such that $\gamma=\gamma_{0} \cup \widehat{\gamma}$ is a compatible path with $\tilde{\gamma}$, which joins from $P_{0}$ to $P_{b}$ along $\gamma_{0}$ and then goes back from $P_{b}$ to $P_{1+}$ along $\widehat{\gamma}$.

Without loss of generality, suppose that $\gamma_{0}$ has arrived at $P_{b}$. Note that $\gamma_{0}$ may meet $P_{b}$ several times, that is, $P_{b}$ is already a reflecting point of $\gamma_{0}$. We first note that Lemma 13 implies that $\Gamma=\gamma_{0} \cup \tilde{\gamma}$ has occupied odd number of crossing points on $D(a)$.

Since $f$ has even number of odd-multiple real roots on $D(a)$, we can take an odd multiple real root $\left(a, x_{1}\right)$ on $D(a)$. By induction assumption of Theorem 25, there exists a small rectangle with $\left(a, x_{1}\right)$ as the center and a small segment of path in the small rectangle, which goes across $\left(a, x_{1}\right)$ from a left endpoint $P_{0}$ on the left side to a right endpoint $P_{1}$ on the right side. Then, $P_{0} \notin D$. Now, we extend the path from $P_{1}$ to the right in the same way as in (A) of case (2(i)). Denote the path by $\gamma_{1}$.

If $\gamma_{1}$ is extended to $P_{b}$, let $\widehat{\gamma}=\gamma_{1}$ and then $\gamma_{0} \cup \widehat{\gamma}_{0}$ is a desired path. Otherwise, $\gamma_{1}$ goes back to $\xi=a$ without touching with $P_{b}$. We can require $\gamma_{1}$ to be extended finally to an odd-multiple real root $\left(a, x_{1}^{\prime}\right)$ with $x_{1} \neq x_{1}^{\prime}$. In fact, if the extension goes back to $\left(a, x_{1}\right)$, it can also be extended to the right by reflecting at $\left(a, x_{1}\right)$ and then repeat the above process. So, if it goes back to $D(a)$, we can always suppose it touches a different odd-multiple real root $\left(a, x_{1}{ }^{\prime}\right)$ on $D(a)$; moreover, we require it to cross $D(a)$ a little with a simple endpoint of $\gamma_{1}$ on the left of $D(a)$.

Denote the path joining with $\left(a, x_{1}\right)$ and $\left(a, x_{1}{ }^{\prime}\right)$ still by $\gamma_{1}$. Then, it has two endpoints of on the left of $D(a)$ and occupies two odd-multiple real roots on $D(a)$. Note that $\Gamma$ occupies odd number of odd-multiple real roots. Then $\Gamma$ and $\gamma_{1}$ have occupied odd number of odd-multiple real roots on $D(a)$.

In the same way as above, take another odd-multiple real root $\left(a, x_{2}\right)$ on $D(a)$. By induction assumption of Theorem 25 , there exists a small rectangle with $\left(a, x_{2}\right)$ as the center and a small segment of path in the small rectangle, which goes across $\left(a, x_{2}\right)$ from a left endpoint $P_{0}{ }^{\prime}$ on the left side to a right endpoint $P_{1}^{\prime}$ on the right side. Then, $P_{0}{ }^{\prime} \notin D$. Now, we extend the path from $P_{1}^{\prime}$ to the right in the same way as in (A) of case (2(i)) to obtain a compatible path $\gamma_{2}$.

We should note that $\gamma_{1}$ plays a role to restrict number of odd-multiple real roots on $D(a)$ which can be taken as a crossing point of $\gamma_{2}$. More precisely, since $\gamma_{1}$ occupied at least two odd-multiple real roots on $D(a)$, a crossing point of $\gamma_{2}$ on $D(a)$ can be chosen in a smaller range.
Below, we analyze several cases of extension of $\gamma_{2}$.

(i) $\gamma_{2}$ does not recur to $D(a)$.

If $\gamma_{2}$ does not go back to $D(a)$, it goes through all $\xi=a_{n}$, and then $\gamma_{2}$ is extended from $\left(a, x_{2}\right)$ to $P_{b}$ in the same way as (A) of case (2(i)).

We should note that $\gamma_{2}$ is allowed to meet with $\gamma_{0} \cup \gamma_{1}$ and $\tilde{\gamma}$ according to the compatibility of paths.

If $\gamma_{2}$ does not attach the path $\gamma_{1}$, then $\gamma_{2}$ is a desired path by taking off $\gamma_{1}$.

Assume that $\gamma_{2}$ attaches $\gamma_{1}$. Since $\gamma_{1}$ is on the left of $\xi=a_{n_{1}}$ with $n_{1}$ being sufficiently big, so $\gamma_{2}$ has no touching point with $\gamma_{1}$ on the right of $\xi=a_{n_{1}}$. Take the last crossing point of $\gamma_{2}$ at $\xi=a_{n_{1}}$ and denote it by $\left(a_{n_{1}}, x_{n_{1}}\right)$.

Then, we drop away $\gamma_{1}$ and the segment of $\gamma_{2}$ which joins from $\left(a, x_{2}\right)$ to $\left(a_{n_{1}}, x_{n_{1}}\right)$. The purpose of dropping $\gamma_{1}$ away is to avoid influence of $\gamma_{1}$ on $\gamma_{2}$ such that $\gamma_{2}$ can only have some touch with $\gamma_{0}$ and $\tilde{\gamma}$; thus, the next extension of $\gamma_{2}$ can be a compatible path.

Then, we extend the segment of $\gamma_{2}$ between $P_{b}$ and $\left(a_{n_{1}}\right.$, $\left.x_{n_{1}}\right)$ to the left. Still denote by $\gamma_{2}$ the segment of $\gamma_{2}$ without confusion. If $\gamma_{2}$ is extended to $D(a)$, then $\gamma_{2}$ is a desired path. Otherwise, the extension goes through all $\xi=a_{n}$ to arrive at $P_{b}$, then we have a closed path which reflects at $P_{b}$ and denote it by $\bar{\gamma}_{1}$. Moreover, $\bar{\gamma}_{1}$ is compatible with $\gamma_{0}$ and $\tilde{\gamma}$.

(ii) $\gamma_{2}$ recurs to $D(a)$.

If $\gamma_{2}$ goes back to $\xi=a$, we assume that it does not touch $\gamma_{1}$ at $\xi=a$ (if so, we can still extend it by reflecting at the touching point and finally $\gamma_{2}$ can reach to an oddmultiple real root on $D(a)$, which does not belong to $\gamma_{1}$ ). Moreover, in the same way as $\gamma_{1}$, we require $\gamma_{2}$ to cross $D(a)$ a little with a simple endpoint on the left of $D(a)$ so that its two endpoints are on the left of $D(a)$. Then, $\gamma_{1} \cup \gamma_{2}$ occupy at least 4 odd-multiple real roots on $D(a)$ and all their endpoints are on the left of $D(a)$. Moreover, $\gamma_{0}, \gamma_{1}, \gamma_{2}, \tilde{\gamma}$ are compatible.

At this stage, we can make $\gamma_{3}$. Using $\gamma_{1} \cup \gamma_{2}$ in place of $\gamma_{1}$ and $\gamma_{3}$ in place of the above $\gamma_{2}$, we can have a similar discussion as above.

Finally, if we have not obtained a desired path, either we obtain another closed path $\bar{\gamma}_{2}$ with $P_{b}$ being a reflecting point, or $\gamma_{1} \cup \gamma_{2} \cup \gamma_{3}$ occupies at least 6 odd-multiple real roots on $D(a)$; moreover, $\gamma_{0}, \gamma_{1}, \gamma_{2}, \gamma_{3}, \tilde{\gamma}$ are compatible.

We1 should note that $\bar{\gamma}_{2}$ is obtained by extension of a segment of the path after dropping $\gamma_{1} \cup \gamma_{2}$. So $\gamma_{0}, \bar{\gamma}_{1}$ and $\bar{\gamma}_{2}$ also follow the compatibility of paths with $\tilde{\gamma}$, and so $\gamma_{0}, \gamma_{1}, \gamma_{2}, \tilde{\gamma}$ are compatible.

If we do not obtain a closed path $\bar{\gamma}_{2}$, we can consider to find $\gamma_{4}$ in the same way as above. Thus, if we do not find a desired path, we either find a closed path $\bar{\gamma}_{2}$ as above or a path $\gamma_{4}$ as $\gamma_{j}, 1 \leq j \leq 3$.

During this process, if we have not obtained a desired path, one of two situations happen: we find one more closed path with $P_{b}$ being a reflecting point or two more odd- 


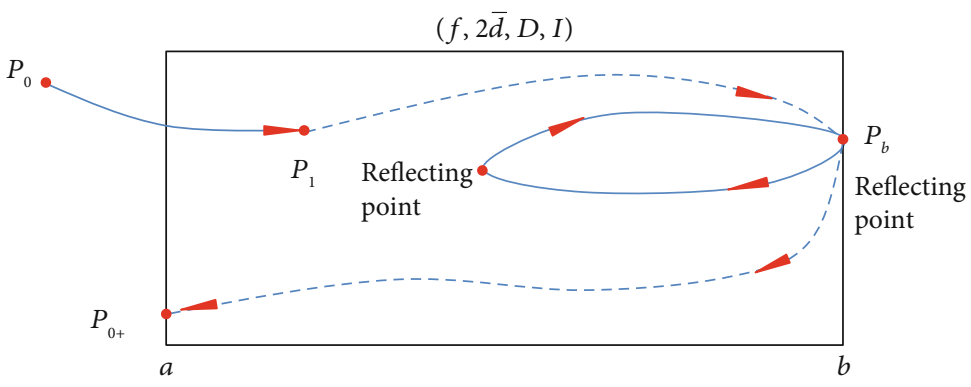

FIGURE 4: Simple extension at even-multiple real roots.

multiple real roots on $D(a)$ are costed by a new path. Moreover, all the paths are compatible.

Also note that we can have at most $\gamma_{1}, \cdots \gamma_{\bar{d}-1}$, which has occupied at least $2 \bar{d}-2$ odd-multiple real roots on $D(a)$. At this stage, if we still have not obtained a desired path, we must obtain a closed path $\bar{\gamma}_{2}$ as above.

(iii) Iteration of the above process

In this way, if the number of closed paths which reflect at $P_{b}$ stops increasing, then more and more odd-multiple real roots on $D(a)$ are costed and after some $n-1$ steps, finally, only one odd-multiple real root on $D(a)$ is left. Then, in $n$-th step, the new path $\gamma_{n}$ cannot recur to $D(a)$, and it then must go to $P_{b}$. If we do not obtain a desired path, the number of closed paths which reflect at $P_{b}$ finally increases by one.

In the same way, if, at some step, the number of closed paths reflecting at $P_{b}$ increases to $\bar{d}-1$, in the next step, the number of closed paths which reflect at $P_{b}$ cannot increase again and the new constructed path, denoted by $\widehat{\gamma}$, has to go back to $\xi=a$. Finally, we obtain some closed paths $\bar{\gamma}_{1}, \cdots, \bar{\gamma}_{m}(m \leq \bar{d}-1)$, which all reflect at $P_{b}$, and a path $\widehat{\gamma}$, which joins with $P_{b}$ and an odd-multiple real root on $D(a)$, such that all path $\gamma_{0}, \bar{\gamma}_{1}, \cdots, \bar{\gamma}_{m}, \widehat{\gamma}, \tilde{\gamma}$ are compatible. Hence, $\gamma=\gamma_{0} \cup \bar{\gamma}_{1} \cup \cdots \cup \bar{\gamma}_{m} \cup \widehat{\gamma}$ is a desired path.

Note that these closed paths $\left\{\bar{\gamma}_{1}, \cdots, \bar{\gamma}_{m}\right\}$ play as a role of bridge to join all the paths $\left\{\gamma_{0}, \bar{\gamma}_{1}, \cdots \bar{\gamma}_{m}, \widehat{\gamma}\right\}$ together to form a compatible path with $\tilde{\gamma}$. In other words, these closed paths have no influence on the continuity of $\gamma$, but they may be important for $\gamma$ to become a compatible path (see Figure 4).

Case of (2(ii)). Note that $B_{2 \bar{d}} \neq \varnothing$. Let $\bar{a}=\inf \left\{\xi \mid \xi \in B_{2 \bar{d}}\right\}$ and $\bar{b}=\sup \left\{\xi \mid \xi \in B_{2 d}{ }^{-}\right\}$. Obviously, $a \leq \bar{a}<\bar{b}<b$ and $\bar{a}, \bar{b} \in B_{2 \bar{d}}$. Suppose $P_{1}=\left(\xi_{1}, x_{1}\right)$ and $a<\xi_{1}<\bar{a}$ or $\bar{b}<\xi_{1}<\mathrm{b}$. Then $f(\xi, x)$ has no $2 \bar{d}$-multiple real root for $\xi \in(a, \bar{a})$ or $\xi \in(\bar{b}, b)$. Then, this case is reduced to that of (2(i)).

Note that $P_{0} \notin D$, then the case that $\xi_{1} \in(\bar{a}, \bar{b})$ cannot happen.

We should note that in a minimal room of real roots $(f, 2 \bar{d}, D, I)$, a path in $D$ can meet at most two $2 \bar{d}$-multiple real roots. This fact is very important for a path to include at most countable even-multiple real roots.

Proof of (3). The result (3) is about the case of odd-degree, the proof is in the same as that of the result (1) by using all induc-

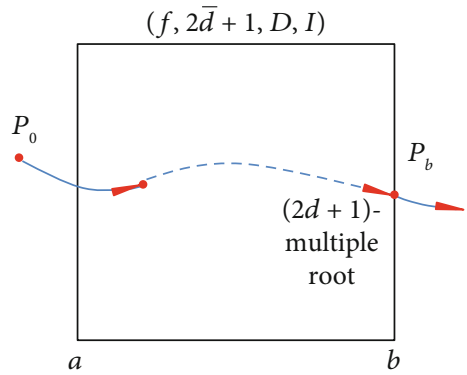

FIGURE 5: Simple extension at odd-multiple real roots.

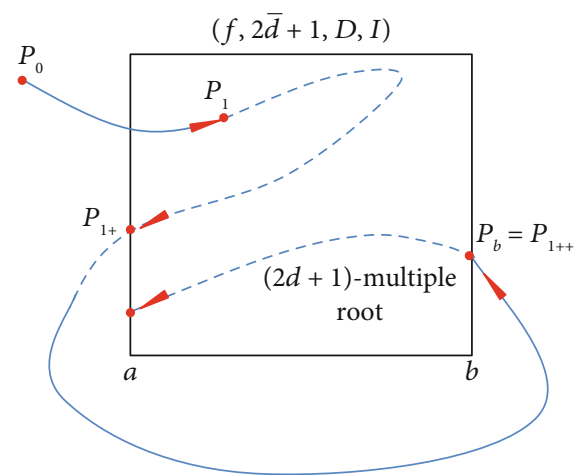

Figure 6: Complicated extension at odd-multiple real roots.

tion assumptions and the result (2(ii)), which is just proven; here, we omit the details.

Proof of (4). Case of (4(i))

In the same way as the proof of (2(i)), the first part of the result can follow by induction assumption and the results (2) and (3); here, we omit the details. See Figures 5-7.

Now, we consider the second part of the result and assume that $\gamma_{0}$ is already extended to $P_{b}$ with $P_{1}=P_{b}$. Below, we consider the two cases:

(i) $P_{b}$ is a crossing point of $\Gamma$

By assumption, $P_{0} \notin D$. Note that the path is extended in $D$ to $P_{b}$. Since $P_{b}$ is a crossing point of $\gamma_{0}$ or $\tilde{\gamma}_{j}$, then $\gamma_{0}$ cannot be extended to go through $P_{b}$ and $P_{1}=P_{b}$ can only be a left endpoint according the compatibility of paths.

If $P_{b}$ is a crossing point of $\gamma_{0}$, then all $\tilde{\gamma}_{j}$ cannot go through $D$. If $P_{b}$ is a crossing point of $\tilde{\gamma}_{j}$, then $P_{b}$ is not a 


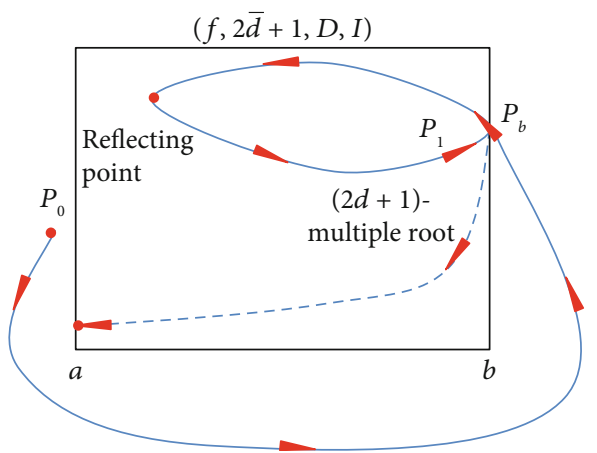

Figure 7: Complicated extension at odd-multiple real roots.

crossing point of $\gamma_{0}$ or $\tilde{\gamma}_{j}$ with $i \neq j$. Anyway, $\Gamma$ occupies even number of crossing points on $D(a)$. Since $f(a, x)$ has odd number of odd-multiple real roots on $D(a)$, we can take an odd-multiple real root on $D(a)$ and a small rectangle with this odd-multiple real root as the center. Then, take a small segment of path crossing this odd-multiple real root in the small rectangle and then extend it to the right in the same way as in the proof of the result (2(i)). If the path reaches to $P_{b}$, then $\gamma_{0}$ can be extended from $P_{b}$ to $D(a)$ along the path; thus, the result is proven. Otherwise, the path does not reach to $P_{b}$ and it goes back to $D(a)$. In the same way as in the proof of (2(i)), we take a new odd-multiple real root on $D(a)$ and repeat the above process. If we do not obtain a desired path from $P_{b}$ to $D(a)$, we have a closed path with $P_{b}$ as a basis point, which is similar to the closed paths $\bar{\gamma}_{j}$ in the proof of (2(i)), where $P_{b}$ is a reflecting point of $\bar{\gamma}_{j}$. Finally, we obtain a desired path from $P_{b}$ to $D(a)$.

Here, we should note that the proof is essentially in the same way as that of the result (2(i)). In fact, $P_{b}$ runs out of 1-multiplicity by a crossing point of $\tilde{\gamma}_{j}$ and remains an even-multiplicity which can be used. So the crossing point $P_{b}$ plays a role as an even-multiple real root, which allows any path arriving at $P_{b}$ to be able to reflect at $P_{b}$.

(ii) $P_{b}$ is not a crossing point of $\Gamma$

Also note that $P_{0} \notin D$ and $P_{0} \neq 6=P_{b}$. If the path is extended to the right to arrive at $P_{b}$, then $P_{b}$ is a simple right endpoint and we need not do any extension in $D$. In fact, $P_{b}$ will become a crossing point in the next extension of $\gamma_{0}$ in a neighbouring room of real roots.

Case of (4(ii)). This case is a special situation of (4(i)) with absence of $\left\{\tilde{\gamma}_{j}\right\}$. By induction assumption, we take an oddmultiple real root on $D(a)$ and extend a small segment of a path crossing this odd-multiple real root to the right. In the same way as the proof of (4(i)), it is easy to find a desired path; here, we omit the details.

Proof of (5). We first prove that, if $P_{1} \notin\left\{P_{a}, P_{b}\right\}$, the path $\gamma_{0}$ can be extended such that it arrives at $P_{a}$ or $P_{b}$. Since $P_{0} \notin D$, then $P_{a}$ or $P_{b}$ is a crossing point of $\gamma_{0}$ and $P_{1} \in \stackrel{\circ}{\text {. }}$
Note that once $P_{a}$ is a crossing point of $\tilde{\gamma}_{j}$, then $P_{b}$ is also a crossing point of $\tilde{\gamma}_{j}$; in this case, $\gamma_{0}$ cannot enter into $D$. So $D \cap \tilde{\gamma}=\varnothing$.

Let $P_{1}=\left(\xi_{1}, x_{1}\right)$. Take a partition

$$
a<a_{n}<a_{n-1}<\cdots<a_{0}<\xi_{1}<b_{0}<\cdots<b_{n-1}<b_{n}<b \text {, }
$$

such that $a_{n} \longrightarrow a$ and $b_{n} \longrightarrow b$. Since $f$ has no $2 \bar{d}+1-$ multiple real root for $\xi \in\left[a_{0}, b_{0}\right]$, by induction assumption and the result (3), we can extend $\gamma_{0}$ such that it arrives at $\xi=a_{0}$ or $\xi=b_{0}$. Denote the segment of extension by $\ell_{1}$.

Now, we consider $\gamma_{1}=\gamma_{0} \cup \ell_{1}$ with an odd-multiple endpoint on $\xi=a_{0}$ or $\xi=b_{0}$ as a new initial endpoint and extend the path $\gamma_{1}$ in $\left[a_{1}, b_{1}\right]$ such that it arrives to $\xi=a_{1}$ or $\xi=b_{1}$. Denote the segment of the extension by $\ell_{2}$.

Then, we can repeat the extension by the result (3) and in the same way as in the proof of (2(i)). If the path is already extended to $\xi=a_{n}$ or $\xi=b_{n}$, we consider $\left[a_{n+1}, b_{n+1}\right]$. Then, it can be extended to reach to $\xi=a_{n+1}$ or $\xi=b_{n+1}$.

We should note that by Lemma 24 , there are at most finitely many $2 \bar{d}$-degree minimal rooms of real roots in the decompositions of rooms on $\left[a_{n}, b_{n}\right]$; thus, the extension of the path on $\left[a_{n}, b_{n}\right]$ includes at most finitely many $2 \bar{d}$ multiple real roots.

Note that the path cannot oscillate to $\xi=a$ and $\xi=b$ more than $2 \bar{d}+1$ times; thus, the path must go along one direction finally. If the path is extended to cross all $\xi=a_{n}$, then, in the same way as in the proof of the result (2(i)), it is extended to $P_{a}$ finally. If the path is extended to cross all $\xi=b_{n}$, it is extended to $P_{b}$.

Case of (5(i)).

(i) $P_{a}$ and $P_{b}$ are crossing points of $\Gamma$

Note that $P_{0} \notin \mathrm{D}$. If $P_{a}$ is a crossing of $\tilde{\gamma}_{j}$, then $P_{b}$ is also a crossing of $\tilde{\gamma}_{j}$, since its endpoints are outside of $D$. In this case, $\gamma_{0}$ cannot enter into $D$. Since $P_{1} \in D$, then $P_{1}=P_{a}$ or $P_{b}$. In this situation, $P_{1}$ can only be a basis point in the extension. If $P_{a}$ is a crossing of $\gamma_{0}$, then $P_{a}$ cannot be a crossing of $\tilde{\gamma}_{j}$, so $P_{b}$ is also a crossing of $\gamma_{0}$. Then, $P_{1}=P_{a}$ or $P_{b}$ can also be a basis point in the extension. Whatever happens, the endpoint of $\gamma_{0}, P_{1} \in D(a) \cup D(b)$ always has outward direction; thus, the result holds.

(ii) Only one of $P_{a}$ and $P_{b}$ is a crossing point of $\Gamma$

Without loss of generality, we assume that $P_{a}$ is a crossing point and $P_{b}$ is not. In this case, neither $P_{a}$ nor $P_{b}$ is a crossing point of $\tilde{\gamma}_{j}, \forall j$. Actually, $D \cap \tilde{\gamma}=\varnothing$, where $\tilde{\gamma}=\cup_{j} \tilde{\gamma}_{j}$.

Since $P_{0} \notin \mathrm{D}$, then $\gamma_{0}$ must enter into $D$ from $P_{a}$. Since we can ignore the presence of $\tilde{\gamma}$, this case is actually the case of (5(ii)); see the proof of the case of (5(ii)) below. Then, we can extend the path to $P_{b}$ such that $P_{b}$ is a right endpoint.

(iii) Neither $P_{a}$ nor $P_{b}$ is a crossing point of $\Gamma$

In the same way as above, we have $D \cap \tilde{\gamma}=\varnothing$. 
Note that $P_{0} \notin \mathrm{D}$. Then, $P_{1}=P_{a}$ or $P_{b}$. Moreover, $\gamma_{0} \cap D$ $(\mathrm{a}, \mathrm{b})=\varnothing$. Thus, this case is also reduced to that of the case (5(ii)).

Case of (5(ii)). If $\bar{d}<d-1$, this result is from the induction assumption of Theorem 25.

Let $\bar{d}=d$. First, take a section $D\left(\xi_{0}\right)$, where $a<\xi_{0}<b$. By the result (4), we have a regular path $\gamma_{0}$ which joins with $P_{0}=P_{a}$ and an odd-multiple left endpoint $P_{1} \in D\left(\xi_{0}\right)$. Then, by the above discussion, it can be extended to $P_{b}$ or $P_{a}$. In the first case, we have a desired path. Otherwise, it arrives at $P_{a}$, the path forms a closed path $\bar{\gamma}_{1}$ with $P_{a}$ as a basis point. In the same way, by the result in the case (4(i)), we have a path $\gamma_{0}$ which joins with $P_{0}=P_{a}$ and an odd-multiple left endpoint $P_{1} \in D\left(\xi_{0}\right)$. Then, by the above discussion, it can be extended to $P_{b}$ or $P_{a}$. By repeating the above process, either we obtain a desired path or we obtain a closed path $\bar{\gamma}_{2}$ with $P_{a}$ as a basis point, which is compatible with $\bar{\gamma}_{1}$.

In this way, if we do not obtain a desired path, then we obtain one more closed path $\bar{\gamma}_{1}$ with $P_{a}$ as a basis point, which is compatible with the previous ones $\bar{\gamma}_{1}, \cdots \bar{\gamma}_{j-1}$. After at most $d$ steps, when we have $d$ number of such closed paths $\bar{\gamma}_{1}, \cdots \bar{\gamma}_{d}$, which are compatible, we can have a path $\gamma_{0}$, which joins with $P_{0}=P_{a}$ and an odd-multiple left endpoint $P_{1} \in$ $D\left(\xi_{0}\right)$. Then, we extend $\gamma_{0}$ from $P_{1}$ to the left and it must go to $P_{b}$ since there is no way for it to go back to $P_{a}$. Moreover, $\gamma_{0}$ is compatible with $\bar{\gamma}_{1}, \cdots \bar{\gamma}_{d}$. Let $\gamma=\gamma_{0} \cup \bar{\gamma}_{1} \cup \cdots \cup \bar{\gamma}_{d}$ , then $\gamma$ is a desired path.

Proof of (6). Case of (6(i))

First note that $\bar{d} \leq d-1$ and $\bar{d}=m$, then it follows that $m \leq d-1$. By induction assumption, Theorem 25 already holds.

By assumption $B_{2 m+1}$ is a nonempty closed set. Let

$$
\bar{a}=\inf \left\{\xi \mid \xi \in B_{2 m+1}\right\}, \bar{b}=\sup \left\{\xi \mid \xi \in B_{2 m+1}\right\}
$$

Then, $\bar{a}, \bar{b} \in B_{2 m+1}$ and $a \leq \bar{a} \leq \bar{b} \leq b$. Let

$$
[\bar{a}, \bar{b}] \backslash B_{2 m+1}=\cup_{j \geq 1}\left(a_{j}, b_{j}\right) .
$$

Then, $f(x, \xi)$ has no $(2 m+1)$-multiple real root for $\xi \epsilon$ $(a, \bar{a}) \cup(\bar{b}, b) \cup\left(\cup_{\mathrm{j} \geq 1}\left(a_{j}, b_{j}\right)\right)$.

Note $D=D_{[a, \bar{a})} \cup D_{(\bar{b}, \bar{b}]} \cup D_{[\bar{a}, \bar{b}]}$, where $D_{[\bar{a}, \bar{b}]}$ is one-way passage of real roots. Below, we consider several cases according to distribution of the endpoints $P_{0}$ and $P_{1}$ about $D$.

By the induction assumption of Theorem 25, there exists a regular path $\widehat{\gamma}$ in $D_{[\bar{a}, \bar{b}]}$ such that $\widehat{\gamma}$ joins with $P_{\bar{a}}$ and $P_{\bar{b}}$. The path $\hat{\gamma}$ is useful in the proof of this part.

\section{(i) $P_{\bar{a}}$ and $P_{\bar{b}}$ are crossing points of $\Gamma$}

By the discussion, we can suppose that there exists $\tilde{\gamma}_{j}$ going through $P_{\bar{a}}$ and $P_{\bar{b}}$ or $\tilde{\gamma}_{j} \cap D_{[\bar{a}, \bar{b}]}=\varnothing, \forall_{j}$. In the first case, $\gamma_{0}$ cannot enter into $D$, so $P_{1} \in D_{[a, \bar{a}]} \cup D_{[\bar{b}, b]}$. Then, by the result of (4(i)), we can extend $\gamma_{0}$ to $D(a)$ or $D(b)$. In the second case, $\gamma_{0}$ must enter into $D$ and go through $D_{[\bar{a}, \bar{b}]}$ with $P_{1} \in D_{[a, \bar{a}]} \cup D_{[\bar{b}, b]}$, then we can extend $\gamma_{0}$ to $D(a)$ or $D(b)$.

(ii) Only one of $P_{\bar{a}}$ and $P_{\bar{b}}$ is a crossing point of $\Gamma$

Without loss of generality, suppose $P_{\bar{a}}$ is a crossing point and $P_{\bar{b}}$ is not. Since $P_{0} \notin D_{[\bar{a}, \bar{b}]}$, then $\gamma_{0}$ must enter into $D$ from $P_{\bar{a}}$. Then, we replace the segment of $\gamma_{0}$ in $D_{[\bar{a}, \bar{b}]}$ with $\tilde{\gamma}$ to extend the path to $P_{\bar{b}}$. Then, we extend the path to cross $P_{\bar{b}}$ to reach to $D(b)$.

(iii) Neither $P_{\bar{a}}$ nor $P_{\bar{b}}$ is a crossing point of $\Gamma$

Since $P_{0} \notin D$, then $P_{1} \notin D_{[\bar{a}, \bar{b}]}^{\circ}$ and $\gamma 0 \cap D_{(\bar{a}, \bar{b})}=\varnothing$. So $P 1$ belongs to $D_{[a, \bar{a}]}$ or $D_{[\bar{b}, b]}$. If $P_{1} \in D_{[a, \bar{a}]}$, the path $\gamma_{0}$ can be extended to $P_{\bar{a}}$ or $D(a)$. If it is extended to $P_{\bar{a}}$, by joining the path $\hat{\gamma}$ with $\gamma_{0}, \gamma_{0}$ can be extended to $P_{\bar{b}}$ and then to $D(b)$. If $P_{1}$ belongs to $D_{[\bar{b}, b]}$, we have similar discussion.

Case of (6(ii)) and Proof of Theorem 25. Now, we are in a position to prove Theorem 25. Recall

$$
\begin{aligned}
& A_{2 d+1}=\{(\xi, x) \mid x \text { is a }(2 d+1) \text {-multiple real root of } f(\xi, x)\}, \\
& B_{2 d+1}=\left\{\xi \mid(\xi, x) \in A_{2 d+1}\right\} .
\end{aligned}
$$

Then, $B_{2 d+1}$ is a closed set.

Let

$$
a_{*}=\min \left\{\xi \mid \xi \in B_{2 d+1}\right\}, b_{*}=\max \left\{\xi \mid \xi \in B_{2 d+1}\right\} .
$$

Then, $a_{*}, b_{*} \in B_{2 \mathrm{~d}+1}$ and $a \leq a_{*} \leq b_{*} \leq b$. Without loss of generality, suppose $a<\mathrm{a}_{*}$ and $b_{*}<\mathrm{b}$. By Lemma 26, there exist a path $\gamma_{l}$ joining with $\left(a_{*}, x_{*}\right)$ and $A(a)$ and another path $\gamma_{r}$ joining with $\left(b_{*}, y_{*}\right)$ and $A(b)$. Let

$$
\left[a_{*}, b_{*}\right] \backslash B_{2 d+1}=\cup_{n} \geq_{1}\left(a_{n}, b_{n}\right) .
$$

Let $x_{n}$ and $y_{n}$ be the $(2 d+1)$-multiple real root of $f\left(a_{n}, x\right)=0$ and $f\left(b_{n}, x\right)=0$. By the result (5(ii)) of Lemma 26, there exists a path $\gamma_{n}: \gamma_{n}(t), \mathrm{t} \in[0,1]$, joining with $\left(a_{n}, x_{n}\right)$ and $\left(b_{n}, y_{n}\right)$.

By rescaling the parameter of the curve $\gamma_{n}$ and suppose $\gamma_{n}: \gamma_{n}(t), t \in\left[a_{n}, b_{n}\right]$. Similarly, suppose $\gamma_{1}: \gamma_{l}(t), t \in\left[a, a_{*}\right]$ and $\gamma_{r}: \gamma_{r}(t), t \in\left[b_{*}, b\right]$.

Moreover, similar to Lemma 19 , it follows that $A_{2 d+1}$ is on the continuous curve $x=-\left(c_{1}(\xi) /(2 d+1)\right)$ determined by $f^{(2 d)}(\xi, x)=0$, so $x_{n}=-c_{1}\left(a_{n}\right) /(2 d+1), y_{n}=-\left(c_{1}\left(b_{n}\right) /(2 d\right.$ $+1)$ ). 


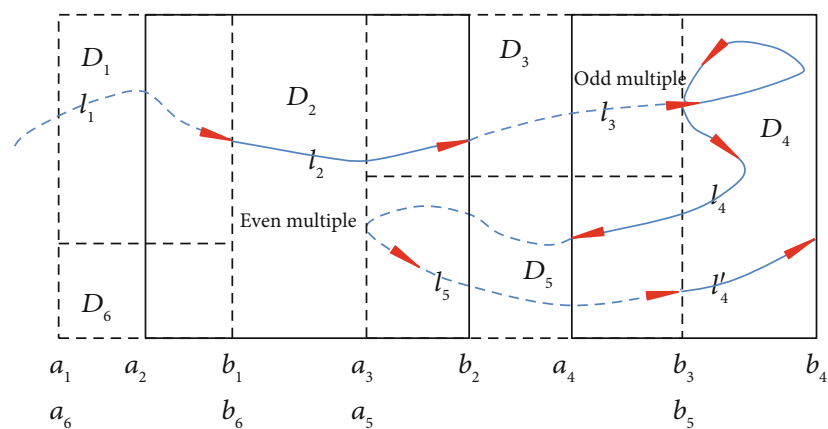

FIGURE 8: Passage of odd-multiple real roots.

Note that $[a, b]=\cup_{n \geq 1}\left[a_{n}, b_{n}\right] \cup B_{2 d+1} \cup\left[a, a_{*}\right] \cup\left[b_{*}, b\right]$. Then, define

$$
\begin{aligned}
& \gamma(t)=\gamma_{n}(t), \quad a_{n} \leq t \leq b_{n}(n \geq 1), \\
& \gamma(t)=\left(t,-\frac{c_{1}(t)}{2 d+1}\right), \quad t \in B_{2 d+1}, \\
& \gamma(t)=\gamma_{l}(t), \quad a \leq t \leq a_{*}, \\
& \gamma(t)=\gamma_{r}(t), \quad b_{*} \leq t \leq b .
\end{aligned}
$$

Then, $\gamma: t \in[a, b] \longrightarrow A$ is a path joining with oddmultiple real roots on $D(a)$ and $D(b)$, respectively. Of course, by rescaling of parameter we can write as $\gamma: t \in[0,1] \longrightarrow A$.

Below we show that $\gamma$ has at most countable reflecting points and basis points.

From the proof of the results (2(ii)) and (4(ii)), the path $\gamma$ includes at most countable number of $2 d$-multiple real roots as reflecting points on each interval $\left[a_{n}, b_{n}\right]$. Moreover, only $\left\{\left(a_{n}, x_{n}\right),\left(b_{n}, y_{n}\right)\right\}$ can be basis points of $(2 d+1)$-multiple real roots, so the path includes at most countable number of $(2 d+1)$-multiple real roots as basis points. Furthermore, the other reflecting points and basis points are lowermultiple real roots and they belong to some segments of the path in some lower-degree passages of odd-multiple real roots. By induction assumption, all reflecting points and basis points on these segments of the path are at most countable. Moreover, these lower-degree passages are also at most countable. Thus, all reflecting points and basis points on the path are also at most countable. For the idea of the proof, we refer to Figure 8.

Proof of Theorem 2. Now, we can prove our main result. By Theorem 25, we only need to consider the case that $f$ has no $(2 d+1)$-multiple real root in $D$. We reduce the case to the special one as in Theorem 25.

Let $a_{0}<a$ and $b<b_{0}$, then $[a, b] \subset\left[a_{0}, b_{0}\right]$. It is easy to see that there exists a real polynomial of $f_{0}(\xi, x)$ of degree $2 d+1$, which depends continuously on $\xi \in\left[a_{0}, b_{0}\right]$, such that $f_{0}(\xi, x)=f_{0}(\xi, x), \xi \in[a, b]$ and both $f_{0}\left(a_{0}, x\right)$ and $f_{0}\left(b_{0}, x\right)$ has a $(2 d+1)$-multiple real root. Then, we use Theorem 25 or the result (5(ii)) of Lemma 26 to $\left(f_{0}, 2 d+1, D_{0}, I_{0}\right)$, where $I_{0}=\left[a_{0}, b_{0}\right]$. There exists a regular path $\left\{\gamma_{0}(t), t \in[0,1]\right\}$, such that $\gamma_{0}(0) \in D_{0}\left(a_{0}\right)$ and $\gamma_{0}(1) \in D_{0}\left(b_{0}\right)$.
Let

$t_{0}=\max \left\{t \in[0,1] \mid \gamma_{0}(t) \in D(a)\right.$ is a crossing point of $\left.\gamma_{0}\right\}$,

$t_{1}=\min \left\{t \in[0,1] \mid \gamma_{0}(t) \in D(b)\right.$ is a crossing point of $\left.\gamma_{0}\right\}$.

Thus, it is easy to see that $\gamma_{0}\left(t_{0}\right) \in D(a)$ and $\gamma_{0}\left(t_{1}\right) \in D(b)$ are odd-multiple real roots of $f$. Moreover, $\left\{\gamma(t)=\gamma_{0}(t) \epsilon\right.$ $\left.D, t \in\left[t_{0}, t_{1}\right]\right\}$ is a path on $D$ with two odd-multiple real roots $\gamma_{0}\left(t_{0}\right)$ and $\gamma_{0}\left(t_{1}\right)$ on $D(a)$ and $D(b)$, respectively.

Recall $T_{P}=\{t \in[0,1] \gamma(t)=P\}$. By construction of $\gamma$, the estimate (11) in Theorem 2 holds obviously. Moreover, for $P \in \gamma$, if $T_{P}{ }^{\#} \geq 2$, then $P$ is a basis point or reflecting point; all these points are at most countable. Thus, Theorem 2 is proven.

Remark 28. From the above proof, we do not know if the path $\gamma$ in Theorem 2 is regular; however, it has no influence on our application.

\section{Data Availability}

No data were used to support this study.

\section{Conflicts of Interest}

The author declares that he has no conflict of interest.

\section{Acknowledgments}

The author would like to thank Professors Jiangong You and Hao $\mathrm{Wu}$ for the many beneficial discussions and help. This study was funded by the National Natural Science Foundation of China $(11871146,11671077)$.

\section{References}

[1] H. W. Broer, H. Hanßmann, and J. You, "Bifurcations of normally parabolic tori in Hamiltonian systems," Nonlinearity, vol. 18, no. 4, pp. 1735-1769, 2005.

[2] H. W. Broer, H. Hanßmann, and J. You, "Umbilical torus bifurcations in Hamiltonian systems," Journal of Differential Equations, vol. 222, no. 1, pp. 233-262, 2006.

[3] L. Corsi and G. Gentile, "Resonant motions in the presence of degeneracies for quasiperiodically perturbed systems," Ergodic Theory and Dynamical Systems, vol. 35, no. 4, pp. 1079-1140, 2015.

[4] J.-X. Xu, "On small perturbation of two-dimensional quasiperiodic systems with hyperbolic-type degenerate equilibrium point," Journal of Differential Equations, vol. 250, no. 1, pp. 551-571, 2011.

[5] J.-G. You, "A KAM theorem for hyperbolic-type degenerate lower dimensional tori in Hamiltonian systems," Communications in Mathematical Physics, vol. 192, no. 1, pp. 145-168, 1998.

[6] V. I. Bakhtin, "Weierstrass-Malgrange preparation theorem in the finitely smooth case," Functional Analysis and Its Applications, vol. 24, no. 2, pp. 86-96, 1990. 
[7] A. O. Gokhman, "The division theorem for diffrerntiable functions (Russian)," Uspekhi Matematicheskikh Nauk, vol. 41, no. 2, pp. 1991-1992, 1986.

[8] M. G. Lassalle, "Une demonstration du theoreme de division pour les functions differentiables (French)," Topology, vol. 12, no. 1, pp. 41-62, 1973.

[9] P. Milman, "The Malgrange-Mather division theorem," Topology, vol. 16, no. 4, pp. 395-401, 1977.

[10] L. Nirenberg, "A proof of the Malgrange preparation theorem," in Lecture Notes in Mathematics, vol. 192 of Proceedings of Liverpool Singularities-Symposium I, pp. 97-105, Springer, Berlin, Heidelberg, 1969.

[11] J. Leray and J. Schauder, "Topologie et équations fonctionnelles," Annales scientifiques de l'École normale supérieure, vol. 51, pp. 45-78, 1934.

[12] J. Mawhin, "Leray-Schauder continuation theorems in the absence of a priori bounds," Topological Methods in Nonlinear Analysis, vol. 9, no. 1, pp. 179-200, 1997.

[13] M. Marden, The Geometry of the Zeros of a Polynomial in the Complex Variables, Volume 3 of Mathematical surveys, American Mathematical Society, 1949.

[14] D. Alekseevsky, A. Kriegl, P. W. Michor, and M. Losik, "Choosing roots of polynomials smoothly," Israel Journal of Mathematics, vol. 105, no. 1, pp. 203-233, 1998. 